\title{
Route duration improvement in wireless sensor and actuator networks based on mobility parameters and flooding control
}

\author{
Daniel G Reina', Sergio L Toral ${ }^{1 *}$, Princy Johnson² and Federico J Barrero ${ }^{1}$
}

\begin{abstract}
Mobility of nodes is one of the main causes of broken links in wireless networks. Although several theoretical models for links and routes selection have been proposed in the literature, so far little effort has been made to apply them to the existing routing protocols. In this article, a decision tree has been incorporated in a reactive routing protocol in order to select the most long-lived routes. The decision tree is based on nodes' mobility parameters typically considered by the theoretical models, such as distance between nodes, relative speed, and nodes' directions. The flooding techniques used within the routing protocols for the routing discovery procedures cause a massive usage of control packets which in turn has negative impact on the performance of the networks. So, an improved flooding control is presented in this article that enhances the performance of the proposed route selection based on a decision tree, in turn reducing overheads and the power consumption caused by the control packets. These two proposed approaches have been implemented over a widely used reactive routing protocol such as Ad Hoc On-Demand Distance Vector (AODV) to obtain performance results using Network Simulator 2 simulation tool. The performances of the proposed approaches have been compared with that of the AODV implementation in terms of general performance and path duration. The simulation results show that the proposed route selection significantly improves the results of AODV.
\end{abstract}

Keywords: intelligent mobile ad hoc networks, route selection, path duration, flooding, AODV

\section{Introduction}

Wireless sensor and actuator networks (WSANs) are wireless networks in which two types of devices can be found, namely, sensors and actuators [1].

The sensor nodes are normally responsible for sensing the environment in which the nodes are deployed, whereas the actuator nodes act as the sink gathering all the data from the sensor nodes. WSANs can be seen as collaborative systems since the nodes exchange data in an ad hoc manner to achieve a common goal [2]. Moreover, the actuators can influence on the behavior of sensors, so the dataflow in those networks is bidirectional. Several limitations influence the performance of WSANs such as limited transmission range of nodes, energy consumption, bandwidth, size, and cost and thus affect their

\footnotetext{
* Correspondence: toral@esi.us.es

${ }^{1} E$. S. Ingenieros, University of Seville, Avda. Camino de los Descubrimientos $\mathrm{s} / \mathrm{n}, 41092$, Seville, Spain

Full list of author information is available at the end of the article
}

deployment [3]. These limitations coupled with the highly dense and mobile nature of the nodes pose a great challenge to the design and management of WSANs [4]. Due to the limited coverage area of nodes in WSANs communications are likely to be multi-hop in such networks. There could be three types of data communications in WSANs in terms of the direction of the data flow [1]: First, Single-sink single-hop communications, where an actuator communicates with a sensor or vice versa using only one hop; second, single-sink multi-hop communications, where a high density of nodes is assumed so that the sensors can communicate with the sink through multiple hops, and finally, multisink multi-hop communications, this is a general scenario where multiple sinks are included. Whenever a source node wants to establish a communication path with a destination node, it must start a discovery route procedure. This discovery procedure depends on the routing protocol used. According to the management of 
the routing tables, the routing protocols are normally categorized as reactive routing protocols, proactive routing protocols, and hybrid routing protocols [5]. The reactive routing protocols are more suitable for high mobile and high-density scenarios due to the required low maintenance for their routing table. Their maintenance requires smaller amount of dataflow among nodes than in proactive routing protocols. As a consequence, reactive protocols incur lower overheads [6-8]. Another classification of routing protocols can be found in [4], where the routing protocols for wireless sensors networks are categorized as data-centric, hierarchical, or location based [9]. Data-centric routing protocols are focused on the interest of the measured information so they work on demand in a reactive way. In hierarchical routing protocols, the nodes are divided into clusters in order to save energy and reduce the overheads. The use of clusters is common in WSANs since the communications are centralized at the actuators which make decisions based on the collected information. The locationbased routing protocols get the positioning information either by Global Positioning System (GPS) or by Received Signal Strength (RSS) [10]. The positioning information is usually used to reduce the overheads by forwarding the data packets directly to the destination nodes, decreasing the number of useless communications [11]. Furthermore, positioning information can also be used to improve the reliability of route selection using GPS [12], or RSS [13].

The routing protocols are normally evaluated in terms of throughput, delay, overhead, delivery ratio, and path duration among others $[7,14,15]$. Although some terms are related to each other, i.e., a high overhead in the network worsens the throughput, the primary objective of designing routing protocols is to improve one of the above-mentioned features [16]. This article is focused on path duration. The path duration in wireless networks gets its importance due to the following reasons:

- Whenever an established route is broken a repair procedure must be carried out in order to re-establish the broken route. Although the recovery procedure depends on the routing protocol used, it normally requires an extra dataflow and consequently extra energy consumption.

- Some applications may require minimum path duration in order to exchange the whole information so the quality of service can be guaranteed [16].

There are some solutions to solve the problem of the time consumed to repair a broken path [14,17]. In multipath routing techniques [14], the routing protocols store more than one route per destination so that alternative routes can be used whenever a breakage of a route occurs. In [17], a bypass mechanism was presented to act against individual broken links along the whole communication path. However, these mentioned solutions do not guarantee path duration since they do not prevent links from being broken in the first place. The breakage of a route can be caused by multiple factors. The main factors which affect the path duration are

- Mobility factors: The relative speed between two nodes, the nodes' directions, and the relative angle between two nodes determine how the overlapping area of the nodes' transmission areas varies and, as a consequence, they have a significant impact on the duration of the links. Additionally, the mobility models also play an important role in path duration since they determine the nodes' movements in the deployed scenario $[17,18]$.

- Positioning factor: The point at which two nodes start communicating with each other influences the link duration and as a result, affects the path duration [18].

- Power factors: The transmission power determines the coverage area of nodes. Notice that the power consumption is a limiting factor in WSANs, and consequently the transmission power is also limited. Besides, if a node with low battery takes part in a communication path, it will put at risk the duration of the path [19].

- Link quality factors: If a noisy environment is considered and a multipath propagation model is used, the path duration can be determined by link quality metrics such as Signal-Noise-Ratio [20] or Bit Error Rate [21]. In high-density scenarios, the interferences among nodes can also affect the performance of the network [22]. The problems in wireless networks related to interference are pointed out and solved in [23] by using a cross-layer design of AODV which selects the best path in terms of signal quality.

- Length of the path: The number of hops also affects the path lifetime. It has been shown that the path duration decreased exponentially with the number of hops [24].

The power transmission of devices is usually fixed by designing factors so the network designer does not have any control over it. It depends on the wireless technology used [25]. On other hand, the link quality factors depend on the environment and it can be unpredictable or changeable. As a consequence, making use of mobility and positioning factors is the easiest way to estimate path duration. In this article, a novel approach for improving path selection based on mobility and positioning factors is proposed. It is aimed at reactive 
routing protocols. A decision tree has been included into a reactive routing like Ad Hoc On-Demand Distance Vector (AODV) [26] in order to accomplish the proposed approach. Additionally, an improved discovery procedure of routes is proposed. It is based on mobility and the number of hops forming the communication routes. The objectives of this discovery procedure are (1) to help to increment the path duration and (2) to reduce the number of control packets used during the discovery procedure.

The remainder of this article is presented as follows: Section 2 details the related study while the definitions of path and link duration in terms of mobility parameters are provided in Section 3. A description of the proposed decision tree is presented in Section 4 and its implementation over AODV routing protocol in Section 5. The optimized discovery procedure is described in detail in Section 6. The experimentation and performance evaluation based on the simulation results of the proposed approaches and their comparisons to AODV are presented in Section 7. Finally, the conclusions of this article can be found in Section 8 .

\section{Related study}

Several models for path duration have been proposed. They can be divided into two groups, namely, experimental models and analytical models. An analytical model for link and path duration in mobile ad-hoc networks has been proposed in [27]. The authors have demonstrated that the link duration between two nodes which take part in the communication depends on the nodes' speed, the distance at which the nodes start communicating with each other, the nodes' coverage area, and the relative angle between the speed vectors of the nodes. A similar analysis based on simulation was presented in [28]. In this case, the routing protocol used was AODV. In [29], the path duration issue was studied considering several mobility models. It was shown that the path duration decreases exponentially with the maximum speed of nodes, and the number of hops. On the other hand, the route duration increases with the radio transmission range. The authors concluded that the path duration for a given mobility model can be characterized by $\lambda_{\text {path }}=\lambda_{0} \frac{h V}{R}$, where $h$ represents the number of hops, $V$ is relative speed, $R$ is the radio transmission range, and $\lambda_{0}$ is determined by the map layout, node density, and other parameters of mobility scenarios. The routing protocols considered for the analysis were Dynamic Source Routing [30] and AODV.

Several approaches have been proposed to use any of the mentioned mobility factors for improving reactive routing protocols. An improved mobility aware AODV was presented in [31]. The approach is intended for improving route selection. In this proposed method, the nodes compute their mobility and identify whether they are suitable to participate in the new route or not. A cross-layer design for reliable route discovery was presented in [32]. The authors proposed to take advantage of the use of the RSS to obtain positioning information. A caution zone is defined at the border of the nodes' transmission ranges, and the nodes located in this caution zone are not taken into account for forwarding operations. The RSS value is also used to estimate link lifetime in [13]. The concept of caution zone is improved in [12] by using GPS information to predict nodes' movements. In this approach, the caution zone is not fixed since it depends on the movements of the neighboring nodes. In [33], the authors proposed to vary the radio transmission range in order to obtain nodes' directions and speeds. However, the variation of the transmission range can have negative impact on other existing communications. The relative angle between two nodes is exploited in [34] for vehicular ad hoc networks. The authors assumed that the nodes are always moving with fixed directions along the lanes. The number of hops forming the path is considered in $[35,36]$; in which a reverse path formation is used to maximize the path duration. In these approaches, the routes with lower number of hops are preferred.

Although several approaches have been proposed, most of them are focused on a few mobility factors. The objective of this article consists of using all the aforementioned mobility factors to build a decision tree in order to improve the route selection in proactive routing protocols. The main contributions of this study are

1 A cross-layer design of AODV which is able to obtain mobility and positioning information taking advantage of an improved neighbor management. This neighboring management is based on the usage of Hello packets. The mobility factors are estimated using RSS measures.

2 Design of a decision tree based on mobility factors, which is proposed to improve the reliability of the path selection. The traditional AODV protocol has significantly been modified in order for the decision tree to be implemented over AODV.

3 An optimized discovery procedure of AODV which reduces the number of control packets used significantly in order to find a communication path between a source node and a destination node. This optimized flooding is based on mobility parameters, and the number of hops that forms a communication path. 


\section{Link and path duration considering mobility factors}

A network is normally modeled as a graph $G=\langle V, E\rangle$, where $V=\{1, \ldots, N\}$ is the set of nodes of the graph and $\mathrm{E} \subseteq \mathrm{VxV}$ is the set of links connecting the nodes. If a channel fading model is not considered, it is reasonable to consider that a communication link between two nodes is established when the distance between the two nodes is less than or equal to the node's transmission range $R$. This model is known in the literature as the disk model [37]. A path between a source node and a destination node $P(S, D)$ is specified as a sequence of nodes $\mathrm{P}(\mathrm{S}, \mathrm{D}) \equiv<\mathrm{N}_{1} \mathrm{~N}_{1, \ldots,}, \mathrm{N}_{\mathrm{k}}>$ where $\mathrm{N}_{1}=\mathrm{S}, \mathrm{N}_{\mathrm{k}}=\mathrm{D}$, $\mathrm{N}_{\mathrm{i}} \neq \mathrm{N}_{\mathrm{j}},\left(\mathrm{N}_{\mathrm{i}}, \mathrm{N}_{\mathrm{i}+1}\right) \in \mathrm{E}$, for $\left.\mathrm{i} \neq \mathrm{j}, 0 \leq \mathrm{i} \leq \mathrm{k}\right)$.

Let $X\left(\mathrm{~N}_{\mathrm{i}}, \mathrm{N}_{\mathrm{j}, \mathrm{t}}\right)$ be an indicator random variable, which has a value of 1 if there is a link between nodes $N_{i}$ and $N_{j}$ at time $t$. Otherwise, the value of $X\left(\mathrm{~N}_{\mathrm{i}}, \mathrm{N}_{\mathrm{j}, \mathrm{t}}\right)$ is equal to 0 .

\subsection{Link duration}

Given two nodes $N_{i}$ and $N_{j}$, at time $t_{1}$, the duration of the link $\left(N_{i}, N_{j}\right)$ is the length of the longest time interval $\left[t_{1}, t_{2}\right]$ during which the two nodes are within the coverage area of each other. Furthermore, the two nodes cannot communicate with each other at times $t_{1}-\sigma$ and $\mathrm{t}_{2}+\sigma$ for $\sigma>0$. So, the link duration is defined as [29]

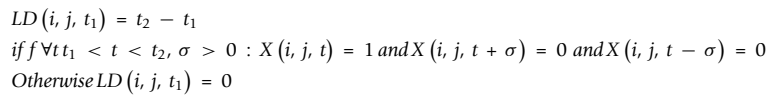

Without losing generality, let us consider $N_{i}$ a static node and $N_{j}$ a mobile node, which is moving at $\vec{v}_{J}$, and both nodes have a transmission range equal to $r$, this situation is illustrated in Figure 1.

The distance $S_{j}$ covered by the node $N_{j}$ before getting out of the coverage area of the node $N_{i}$ at $t_{2}$ via the point $B$ is

$$
S_{j}=r-d_{(i, j)}
$$

The time spent by $N_{j}$ to cover $S_{j}$ is

$$
\operatorname{LD}\left(\mathrm{N}_{\mathrm{i}}, \mathrm{N}_{\mathrm{j}}, \mathrm{t}_{2}-\mathrm{t}_{1}\right)=\frac{S_{j}}{v_{j}}
$$

where $v_{j}$ is the speed at which the node $N_{j}$ moves.

Moreover $d_{(i, j)}$ at $t_{1}$ is a function of $\beta_{(i, j)}$

$$
d_{(i, j)}=r-\left[\left(t_{1}-t_{0}\right) \cos \beta_{(i, j)}\left|v_{j}\right|\right]
$$

where $t_{0}$ is the time at which $N_{j}$ get into the coverage area of $N_{i}$ via the point $A$. So, $\mathrm{LD}\left(\mathrm{N}_{\mathrm{i}}, \mathrm{N}_{\mathrm{j}}, \mathrm{t}_{2}-\mathrm{t}_{1}\right)$ can be calculated as follows

$$
\operatorname{LD}\left(\mathrm{N}_{\mathrm{i}}, \mathrm{N}_{\mathrm{j}}, \mathrm{t}_{2}-\mathrm{t}_{1}\right)=\frac{r\left[1-\left[\left(t_{1}-t_{0}\right) \cos \beta_{(i, j)}\left|v_{j}\right|\right]\right]}{v_{j}}(1)
$$

The maximum LD occurs when the two nodes start communicating with each other in the limit of their coverage areas via the point $O$, with $\beta_{(i, j)}=0$, and reaching the point $C$ at $t_{2}$. This maximum LD is illustrated in Figure 2 as a function of $\beta$.

\subsection{Path duration}

Given a path $\mathrm{P}(\mathrm{S}, \mathrm{D}) \equiv<\mathrm{N}_{0}, \mathrm{~N}_{1, \ldots}, \mathrm{N}_{\mathrm{k}-1}>$, with $k$ nodes, at time $t_{1}$, the path duration is the length of the longest

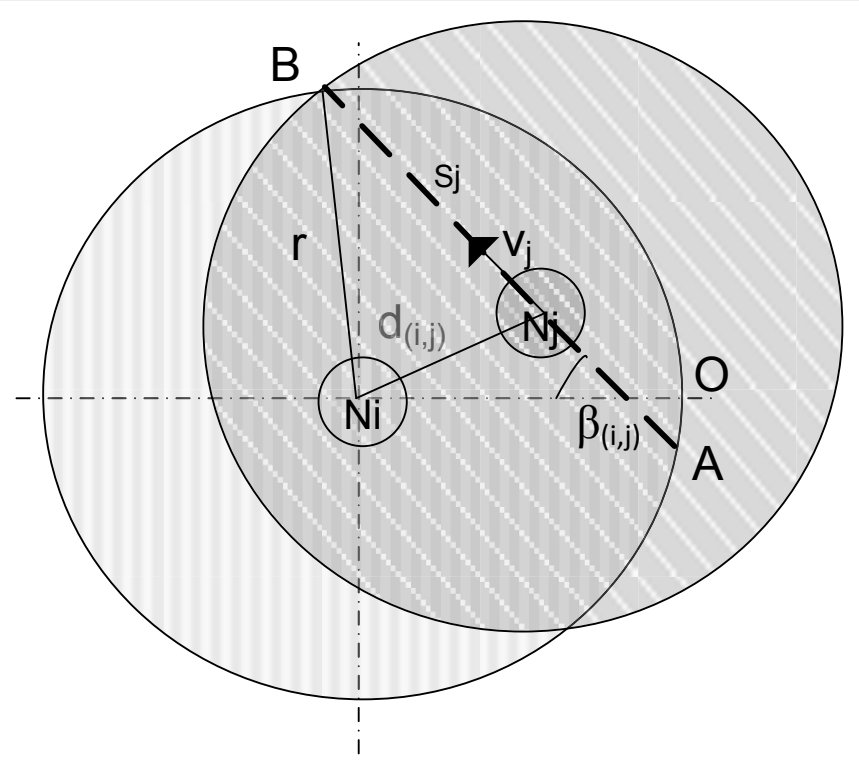

Figure 1 Link duration scenario. 


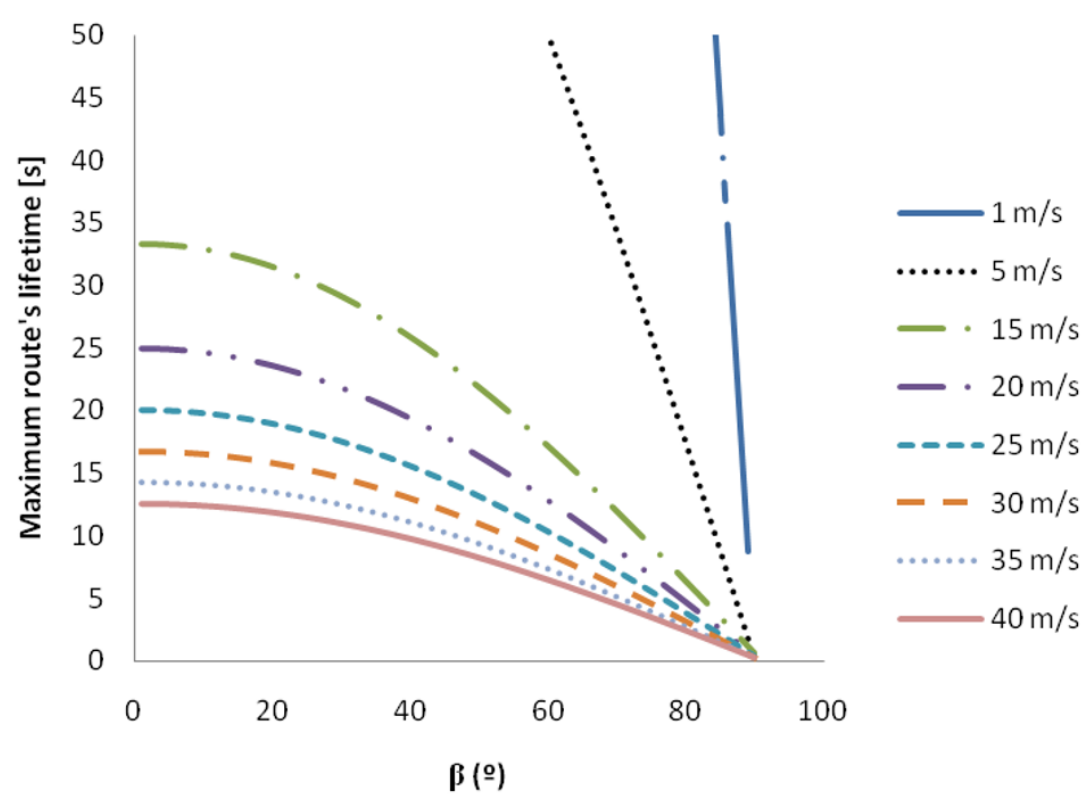

Figure 2 Maximum route's duration.

time interval during which each of the $k-1$ links among the nodes exist. Moreover, at time $t_{1}-\sigma$ and $t_{2}-\sigma$, one or more links are already broken. Thus, the duration of the path is limited by the duration of the links along the whole path. In fact, the path lifetime is defined by the minimum link duration along the path. So, the path duration is

$$
\operatorname{PD}\left(\mathrm{P}, \mathrm{t}_{1}\right)=\min _{1 \leq \mathrm{u} \leq \mathrm{k}-1} \operatorname{LD}\left(\mathrm{N}_{\mathrm{u}}, \mathrm{N}_{\mathrm{u}+1}, \mathrm{t}_{1}\right)
$$

\section{Decision tree building based on nodes' mobility parameters}

\subsection{Neighbor management}

The key idea is to use the hello packets to collect information related to the movements of the neighboring nodes. Although AODV already implements a neighboring table, in this study, an improved version that includes the positioning and direction information is used. The nodes periodically measure the RSS value of those nodes within their coverage areas, even if there is not any active communication. In AODV, already hello packets are used to ensure connectivity. Therefore, the proposed improved neighbor management takes advantage of this dataflow to collect extended information related to nodes' relative positions and nodes' relative mobility.

\subsection{Mobility assumptions}

The proposed decision tree is based on several assumptions which take into consideration the link analysis discussed in Section 3, Equation (1). Notice that the path duration is limited by the shortest link along the whole communication path, so this shortest link determines the quality of the selected route in terms of lifetime. These assumptions are further explained below:

\section{Positioning assumptions}

(1) When a neighboring node is located on the border of a target node's coverage area, it is more likely to get out of the transmission range and, consequently, it is more likely to break an established link.

(2) When a neighboring node is located closer to a target node, it is less likely to get out of the coverage area. Consequently, it is less likely to break an established link.

\section{Speed assumptions}

(1) High relative speed is a negative condition for link duration, since the distance between two nodes is likely to get longer quickly.

(2) When relative speed between two nodes is low they are likely to stay closer for a longer period of time. Consequently, they may form a lasting communication path.

\section{Direction assumptions}

(1) An established link is more likely to be broken when nodes are moving away from each other.

(2) By contrast, when the two nodes are mutually approaching, the established link between them is likely to keep active. 


\subsection{Classification of mobility parameters}

Mobility parameters for building the decision tree are considered as follows:

\section{Distance between two nodes}

- Long distance, whenever the distance between two nodes is higher or equal to $200 \mathrm{~m}$. This distance is called cut off distance. It is illustrated in Figure 3. Otherwise, it is considered as a short distance. Note that the transmission range is considered to be 250 m. It is shown in Figure 4.

\section{Relative speed}

- High relative speed, whenever it is higher or equal to $15 \mathrm{~m} / \mathrm{s}$, so the limiting relative speed is $15 \mathrm{~m} / \mathrm{s}$. The cases 1 and 2 in Figure 3, and the cases 5 and 6 in Figure 4 represent this condition.

- Otherwise, the relative speed is considered as a low speed. It is shown in the cases 3 and 4 in Figure 3 and in the cases 7 and 8 in Figure 4.

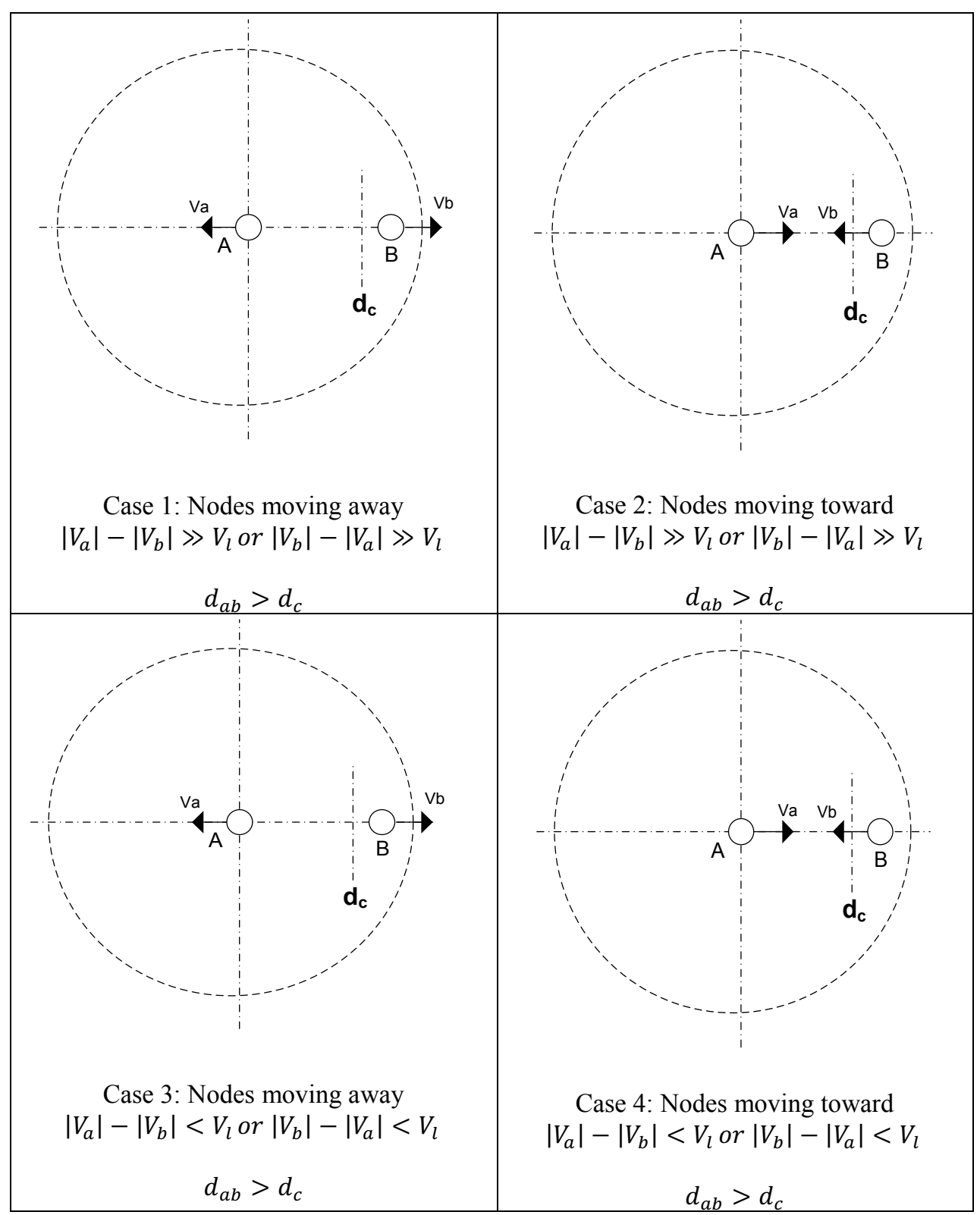

Figure 3 Considered scenarios for different mobility parameter values (II). 


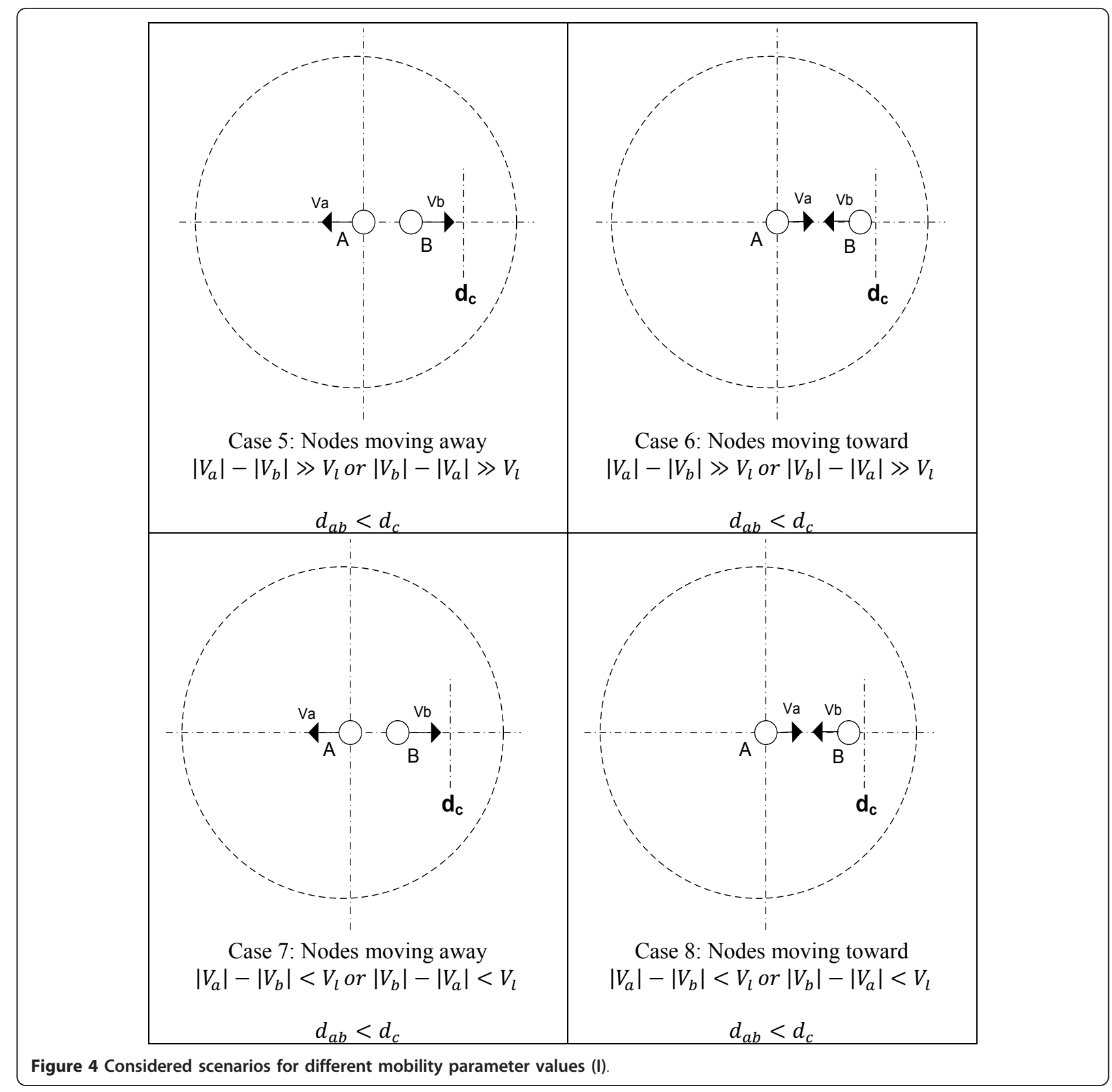

\section{Relative direction}

- Away direction, whenever the nodes are moving away. It is illustrated in Figure 3 cases 1 and 3, and in Figure 4 cases 5 and 7.

- Otherwise, it is considered as closer direction. It is shown in Figure 3 cases 2 and 4, and in Figure 4 cases 6 and 8 .

Table 1 illustrates the different cases included in the decision tree. The column labeled as Link Metric (LM) shows the resulting numeric value associated with the duration of the link between two nodes. A metric of
Table 1 Decision tree table

\begin{tabular}{lllllll}
\hline Distance $(\mathbf{m})$ & Speed $(\mathrm{m} / \mathbf{s})$ & Direction & LM & Case & $\boldsymbol{T}_{\max }(\mathbf{s})$ & $\boldsymbol{T}_{\min }(\mathbf{s})$ \\
\hline Long & High & Away & 0 & Case 1 & 5 & 1.6 \\
Long & High & Closer & 2 & Case 2 & 16.6 & $\mathrm{a}$ \\
Long & Low & Away & 1 & Case 3 & 10 & 3.3 \\
Long & Low & Closer & 4 & Case 4 & 33 & $\mathrm{a}$ \\
Short & High & Away & 1 & Case 5 & 8.3 & 1.6 \\
Short & High & Closer & 2 & Case 6 & 15 & 3.1 \\
Short & Low & Away & 2 & Case 7 & 16.6 & 3.3 \\
Short & Low & Closer & 3 & Case 8 & 30 & 16.6 \\
\hline
\end{tabular}

${ }^{a}$ It depends on the arrival angle. 
four corresponds to the maximum link duration scenario is described in Section 3, while a value of zero corresponds to a bordering node moving away. The minimum and maximum link's lifetime value for each LM are also included in Table 1.

Figures 3 and 4 illustrate the different cases considered for building the decision tree. The limiting relative speed is denoted by $V_{1}$, and the cut off distance is denoted by $d_{\mathrm{c}}$.

For each link participating in the discovery procedure of routes, the LM is calculated. The path metric is defined as the minim LM along the complete communication path. $\operatorname{PM}\left(P, t_{1}\right)=\min _{1 \leq u \leq k-1} L M\left(N_{u}, N_{u+1}, t_{1}\right)$.

\section{Implementation over AODV routing protocol 5.1 Overview of the AODV routing}

AODV is a reactive routing protocol that uses routing tables with one entry per destination. AODV is based on broadcast route discovery mechanism. Three types of packets are used in AODV:

- The Request packets (RREQs): RREQs are normally used whenever a source node wants to start communicating with a destination node; it floods the network with request packets. An RREQ is forwarded until it reaches the destination node or a node which has a route to communicate with the destination node. For each retransmission, the number of hops is incremented. Moreover, RREQs are used to form the direct path between the source and the destination nodes.

- The reply packets (RREPs): RREPs are used to respond to the RREQs, and to form the reverse path. To maintain the most recent routing information among nodes, the concept of destination sequence number is used [38].

- The error packets (RRERs): RRERs are sent whenever a node detects a connectivity failure in the network. It is flooded to all the nodes which form the communication path in order to remove the unreachable routes from their routing tables.

- Hello packets (HELLOs): HELLOs are used to manage local connectivity. The information on the neighbors collected by HELLO packets is stored in a table of neighbors.

AODV is recommended for dynamic topologies, and it exhibits a low overhead compared with other reactive routing protocols [6].

\subsection{Building a decision tree for the AODV routing}

A decision tree has been included in the routing management of AODV. Several modifications have been carried out on the traditional AODV routing protocol in order to facilitate the implementation of the decision tree over the protocol. These modifications are

- The RSS value is measured by means of a crosslayer design of AODV [21,39]. The two-ray propagation model has been considered (2), where $P_{\mathrm{t}}$ is the transmission power, $h_{\mathrm{r}}$ and $h_{\mathrm{t}}$ are the heights of the transmitter and receiver antennas, respectively, $G_{\mathrm{t}}$ and $G_{\mathrm{r}}$ are the antenna gains, and the $L$ is the system loss. The RSS value of each neighboring node is sensed periodically using the hello packets. The RSS values are stored in the table of neighbors. In addition, the RSS value is also measured in the discovery procedure using the routing packets $R R E Q$, and RREP in order to have the newest information of the neighboring nodes before forming a new route.

$\operatorname{Pr}(d)=\frac{h_{r}^{2} h_{t}^{2} G_{r} G_{t} P_{t}}{d^{4} L}$

- The relative direction between two nodes is calculated using two consecutive measures of the RSS value (3). Again, this information is stored by the neighbors in their respective tables. Notice that if $\Delta$ RSS $\left(t_{i}\right)>0$ means that the nodes are getting closer. Otherwise, the nodes are moving away from each other.

$\Delta R S S\left(t_{i}\right)=R S S\left(t_{i}\right)-R S S\left(t_{i-1}\right)$

- The distance between two nodes is estimated using the RSS value, since a very precise calculation is not necessary [10]. For noisy environments, this estimation can be improved by using a GPS.

- The node's speed is sensed locally at each node, and is incorporated into both the control messages RREQ and RREP [31]. The modified request and reply packets are shown in Figures 5 and 6 , respectively.

- The relative speed between two nodes $A$ and $B$ is calculated as follows:

$|V r|=|V a|-|V b|$

- The LM of Table 1 has also been included in the RREP and RREQ packets. The LM is assigned either when an RREQ is received or an RREP is sent. If the assigned LM is lower than the LM included in the control packet, the LM will be updated. As a consequence, the LM included in the control packet is 


\begin{tabular}{|c|c|c|c|c|c|c|c|}
\hline Type & $\mathrm{J}$ & $\mathrm{R}$ & $G$ & $\mathrm{D}$ & $\mathrm{U}$ & Reserved & Hop Count \\
\hline \multicolumn{8}{|c|}{ RREQ ID } \\
\hline \multicolumn{8}{|c|}{ Destination IP address } \\
\hline \multicolumn{8}{|c|}{ Destination sequence number } \\
\hline \multicolumn{8}{|c|}{ Source IP address } \\
\hline \multicolumn{8}{|c|}{ Source sequence number } \\
\hline \multicolumn{8}{|c|}{ Node's speed [m/s] } \\
\hline \multicolumn{8}{|c|}{ Path metric } \\
\hline
\end{tabular}

Figure 5 Modified request packet

always the minimum found along the path, which can be the inverse path in the case of RREP packets or the direct path in the case of RREQ packet.

\subsection{Route formation}

The discovery procedure of AODV has been modified in order to include the decision tree. Traditional AODV route selection is based on destination sequence and number of hops. The destination sequence provides an idea of the newness of the discovered routes, and the newest route is always selected. If two routes have the same destination sequence, the route with lesser number of hops is selected. These criteria ensure the avoidance of endless loops in route selection process [40]. In the proposed approach, the path metric is added to the aforementioned criteria in order to determine the quality of the routes. If two routes have the same destination sequence and a lower or equal number of hops, mandatory criteria to avoid loops, the path metric will be used to break the equality. Moreover, the response of AODV to RREQ packets in the destination node has been modified to have more chances of finding a better route. In AODV, only the first request received by the destination node is attended and the remainders of the received requests are discarded. This is acceptable in AODV since it does not have any other criterion to discern the quality of the incoming routes. In the proposed approach, however, all the incoming requests from the source node that improve the reliability of the target route are taken into consideration. That is, an RREP packet is sent back to all of those RREQ packets. Notice that the destination sequences of these routes are higher so they will be always accepted. To illustrate the proposed approach a general scenario in which a source node $S$ wants to communicate with a destination node $D$ is shown in Figure 7, where there are two possible routes namely, (a) $S-A-B-D$, and (b) $S-C-E-D$. The metrics for each link are represented in Figure 7. As mentioned earlier, the path metric is defined by the minimum LM along the communication path, as illustrated in Figure 8. The routes' path metrics for the proposed scenario are (a) $\mathrm{PM}=1$, and (b) $\mathrm{PM}=2$ (Figure 8 ). Considering that the routes are found in $t_{1}$, and $t_{2}$, respectively, only the route (a) is attended in AODV. In the proposed approach, the route (b) could also originate an RREP in the destination node in case the included path metric is better. Although it may increment the routing load for sending more reply packets, it also helps to increase path duration.

The routing tables of AODV have been modified in order to include the path metric of the routes. For each entry in the routing table, the following data are stored, as shown in Figure 9.

\begin{tabular}{|c|c|c|c|c|c|}
\hline Type & $\mathrm{R}$ & A & Reserved & Prefix Sz & Hop Count \\
\hline \multicolumn{6}{|c|}{ Destination IP address } \\
\hline \multicolumn{6}{|c|}{ Destination sequence number } \\
\hline \multicolumn{6}{|c|}{ Source IP address } \\
\hline \multicolumn{6}{|c|}{ Lifetime } \\
\hline \multicolumn{6}{|c|}{ Node's speed [m/s] } \\
\hline \multicolumn{6}{|c|}{ Path metric } \\
\hline
\end{tabular}

Figure 6 Modified reply packet 


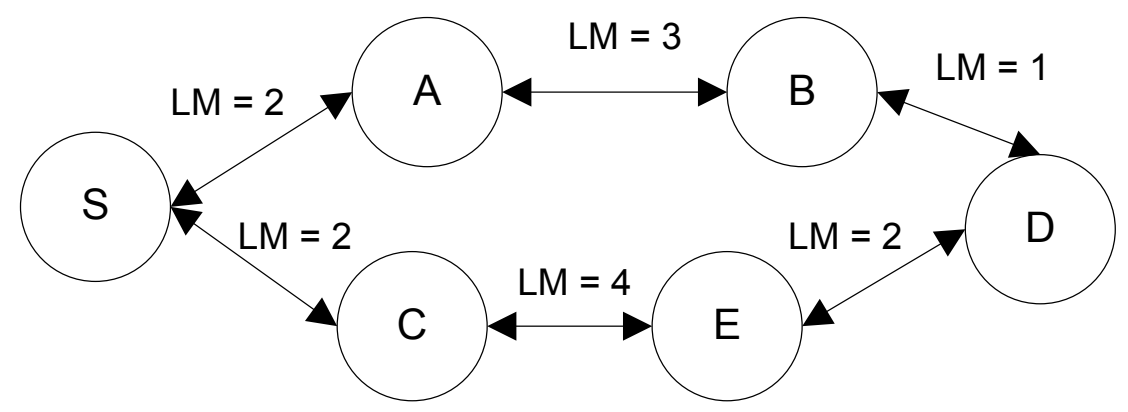

Figure 7 Determining the $L M$ in a general scenario.

\section{Optimized discovery procedure}

The discovery procedure of AODV is based on flooding REQUEST packets. These control packets are flooded and forwarded throughout the network until one or more of them reach the destination node. The main drawbacks of flooding discovery procedure are (1) implosion caused by duplicated control messages, (2) overlap which occurs whenever two nodes sensing the same area send similar packets to the same neighboring node, and (3) resource blindness that consumes large amount of energy without considering individual nodes' [4]. As a result, this discovery procedure may cause a massive overhead in the network since no control over the distribution of the control packets exists. The overhead worsens the performance of the network and hence must be avoided as far as possible. In this article, an optimized discovery procedure is presented, based on the mobility parameters and the number of hops which forms a communication path. The proposed discovery procedure works as described below.

- Since routes with lower path metric last only for a short time, the overhead caused by these paths do not compensate for the short duration of their paths. In the optimized discovery procedure, whenever a node receives a request packet with low LM, it will not forward the request packet. As a result, short lifetime paths do not take part in the communications. For example, in Figure 10, the nodes $E$ and $F$ will not participate in the discovery procedure because the LM in both cases is equal to zero, which is the lowest metric possible. This approach favors the usage of durable path.

- In addition to the flooding control based on mobility factors, the number of hops can also be limited in order to reduce overhead. This is possible as long as the type of scenario is known to the system. The average number of hops which form the communication routes can be estimated from previous simulations. A conservative control of number of hops is proposed. In the proposed flooding control scheme, whenever a node receives a request packet which has been forwarded $K+1$ times, where $K$ is the average number of hops previously calculated by simulation, it will not forward the received request packet any more. This proposed flooding control avoids control packets being forwarded more than it is necessary. Notice that this technique has to be implemented with care since it directly affects the performance of AODV. The improved discovery procedure can easily be added to the proposed AODV based on the decision tree, resulting in a very significant improvement of the route selection scheme of AODV.

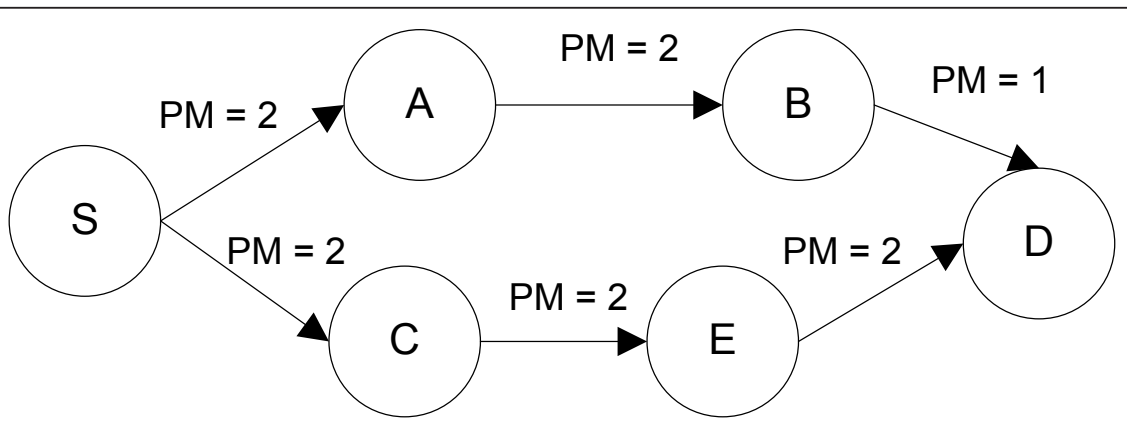

Figure 8 Updating path metric. 


\begin{tabular}{|c|c|c|c|c|c|}
\hline $\begin{array}{c}\text { Source } \\
\text { address }\end{array}$ & $\begin{array}{c}\text { Destination } \\
\text { address }\end{array}$ & $\begin{array}{c}\text { Destination } \\
\text { sequence } \\
\text { address }\end{array}$ & $\begin{array}{c}\text { Hop } \\
\text { count }\end{array}$ & Path metric & Lifetime \\
\hline
\end{tabular}

Figure 9 Routing table fields.

\section{Performance evaluation}

The proposed approaches have been evaluated using simulation and their performances compared with that of the traditional AODV protocol. The network simulator 2 (ns-2.35) has been used to implement the proposed approaches [41].

\subsection{Evaluation metrics}

The following metrics were considered to compare the performance of the routing protocols [14-16]. These metrics are divided into two groups: (1) network performance and (2) path duration.

The metrics included in the network performance group are

- Throughput: Total data packets successfully received by their destinations divided by the simulation time.

- Packet Delivery Fraction (PDF): PDF is the ratio between the number of packets originated by the application layer sources and the number of packets received by the destinations. It will describe the loss rate that will be seen by the transport protocol. A high value of PDF is desired.

- Normalized Routing Load (NRL): The number of routing packets transmitted per data packet delivered at the destination. A low value of NRL is desired since it implies overhead in the network and consequently energy consumption. A low value of this metric is always desired.
- Average end-to-end delay (E2E): This includes all possible delays, caused by buffering during routing discovery latency, queuing at the interface queue, and retransmission delays at the MAC, propagation and transfer times.

- Request packets (RREQ): The number of request packets is important for two reasons: (1) they determine the routing load since they are massively used during the discovery procedure, and (2) the RREQs are sent whenever a broken link occurs as a result of a source node trying to reestablish the broken path. For these reasons, a low number of request packets is also desired. Although this metric has been included in the general performance group it also helps to determine the path durations.

The metrics related to path duration are

- Broken Links (BL): The total number of broken links during the simulation time. This is the most important metric in this study because the primary objective of the proposed approaches is to lengthen the lifetime of the routes.

- Time between errors (TBE): TBE is the time between two consecutive broken links in the actuator nodes. This metric provides an idea about how often the broken links occur referred to the actuator nodes. A high value of this metric is always desired.

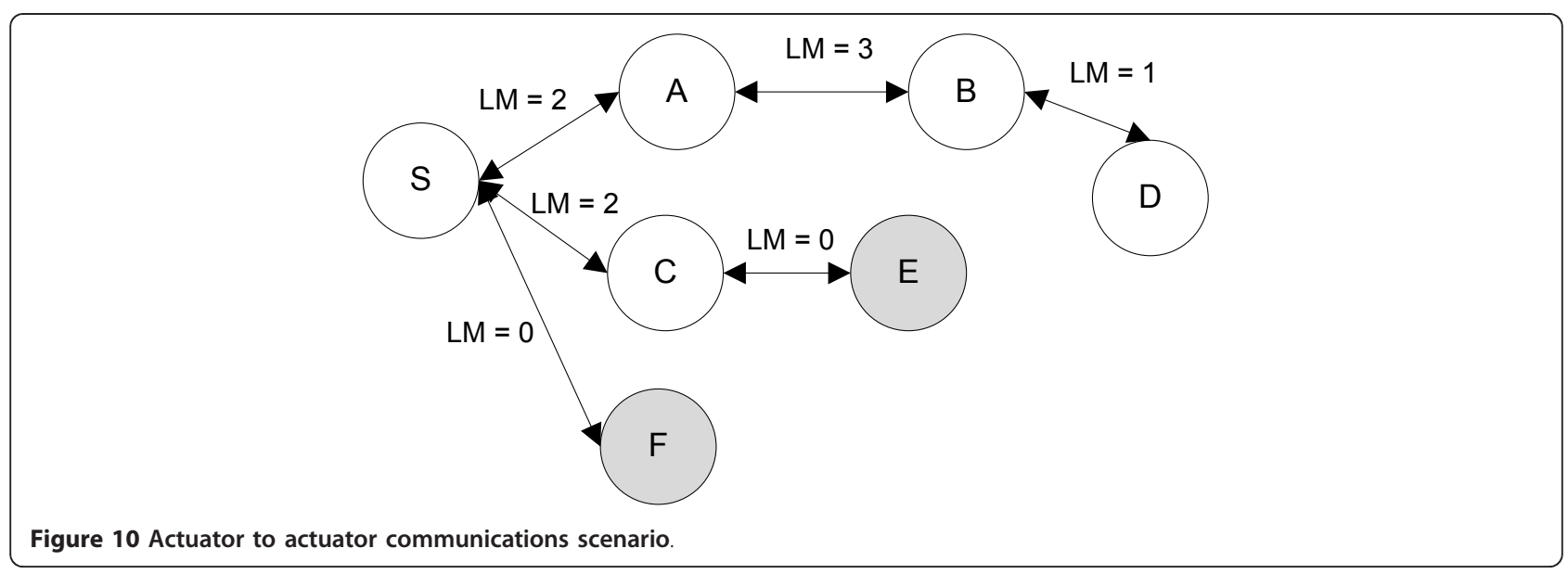




\subsection{Evaluation conditions}

The proposed approaches have been evaluated under two types of conditions: (1) congestion and (2) mobility. In the congestion evaluation, the number of flows between the pairs source-destination has been increased from 10 till 60 connections. This study evaluates the performance of the proposed approaches under different congestion levels. On the other hand, the mobility analysis evaluates the proposed approaches under different mobility conditions. The maximum node's speed has been varied from 10 to $50 \mathrm{~m} / \mathrm{s}$. Notice that the proposed approaches are intended for high mobility scenarios as they take advantage of the obtained positioning and speed measurements through a cross-layer design.

\subsection{Simulation parameters}

The general parameters of simulation are given in Table 2 .

The widely used waypoint mobility model has been used to generate random movements [42]. In this model, a node moves from its current position to a new position by selecting a random direction and a random speed. Whenever the node reaches its destination, it stops during a pause time and chooses a new destination point. The pause time for mobile sensors has been fixed as 0 , which means all nodes are always moving in the entire simulation period. The mobility generator setdest has been used to implement the waypoint

\section{Table 2 Simulation parameters}

\begin{tabular}{ll}
\hline Simulation parameter & Value \\
\hline Number of sensors & 50 \\
Number of actuators & 9 \\
Area, $m \times m$ & $1500 \times 1500$ \\
Active source nodes & {$[10-60]$} \\
MAC protocol & 802.11 \\
Propagation model & Two-ray ground \\
Bit rate & $2 \mathrm{Mb} / \mathrm{s}$ \\
Carrier frequency & $914 \mathrm{MHz}$ \\
Maximum transmit power & $0.282 \mathrm{~W}$ \\
Correct receive threshold & $3.652 \times 10^{-10} \mathrm{~W}$ \\
Threshold to avoid collisions & $1.559 \times 10^{-11} \mathrm{~W}$ \\
Interface queue type & Queue/DropTail/PriQueue \\
Maximum number of packets in queue & 50 \\
Traffic pattern & CBR \\
Application & UDP \\
Packets size & 512 bytes \\
Nodes' positions & Random \\
Transmission range & $250 \mathrm{~m}$ \\
Mobility model & Waypoint \\
Nodes' speed & {$[1-30]$ to [1-50] m/s } \\
Pause time & $0 \mathrm{~s}$ \\
Simulation time & $1000 \mathrm{~s}$ \\
Warm up period & $100 \mathrm{~s}$ \\
\hline & \\
\hline &
\end{tabular}

mobility model [41]. The initial positions of the nodes are random. The traffic generator cbrgen.tcl, which is provided with ns-2 [41], has been modified in order to develop the following simulation scenarios.

\subsection{Simulation scenarios}

The proposed approaches have been tested against three different connectivity scenarios. These scenarios represent the majority of possible situations in WSANs. In all these scenarios, the actuators are considered as static nodes, while the sensor nodes are considered as mobile nodes. The three scenarios are represented in Figures 11,12 , and 13. The actuators are denoted by black rhombuses, the sensors are denoted by white circles, and the dashed arrows represent the communication routes among nodes.

- Scenario A: Actuator-to-actuator communications (A2A). In this scenario, the distance between two actuators is $300 \mathrm{~m}$. This distance is likely to be covered just with a few hops; so, the number of hops is likely to be less than 3 . This scenario is focused on testing multi-hop communications among actuators, and is represented in Figure 11.

- Scenario B: Actuator-to-sensors communications (A2S). In this scenario, the communications are carried out between one actuator and one sensor. The distance between two actuators is again $300 \mathrm{~m}$. The number of hops is variable since it depends on the proximity of the selected sensors. Moreover, the mobility of nodes which take part in the communications is likely to be higher than in the previous scenario. Notice that in the previous scenario there were always two static nodes forming the communications paths. This scenario is illustrated in Figure 12.

- Scenario C: Sensors-to-actuator communications (S2A). In this scenario, the source nodes are the sensors, while the destination nodes are the actuators. Multiple connections can be established with one actuator, so the actuators act as the sinks of the communications. This scenario is represented in Figure 13.

In all the aforementioned scenarios, the number of connections among the nodes is varied from 10 up to 60 connections in steps of 5 connections. These connections start randomly during the warm up period, and they finish at the end of the simulation time. The source-destination node pairs are randomly selected.

\subsection{Optimization of the discovery procedure}

In order to assign a value to the $K$-parameter of the proposed flooding control, it is necessary to know the 


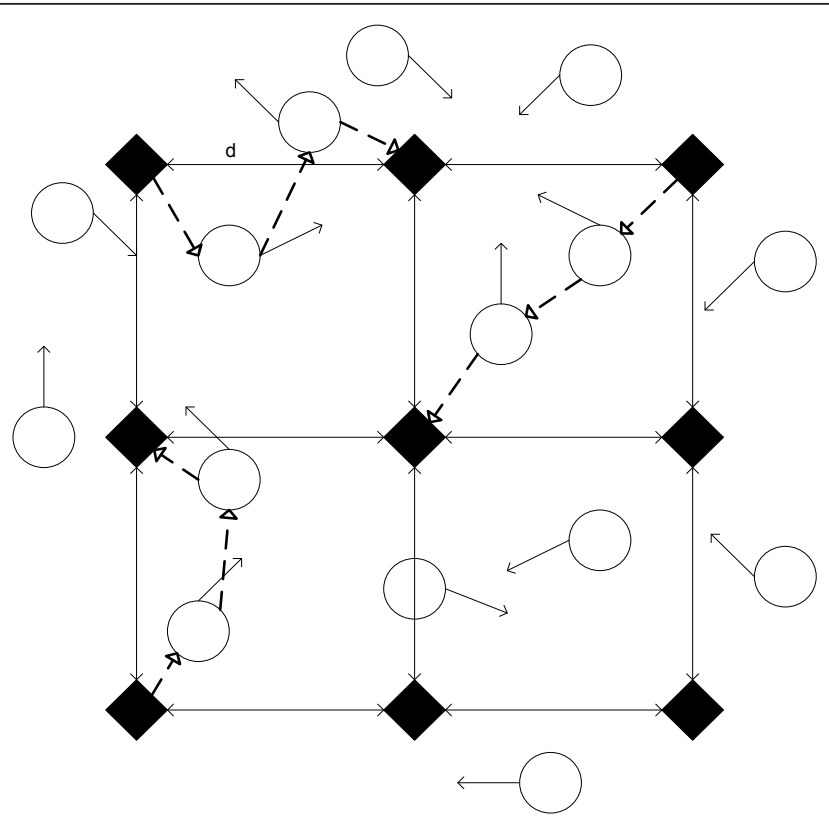

Figure 11 Actuator to sensors communications scenario.

average number of hops which form the communication routes in every proposed simulation scenario. Figure 14 shows the average number of hops for each scenario, which were obtained by simulation.

As it may be expected that the scenario A (A2A communication) requires less number of hops than the other two scenarios, while the scenario C (S2A communication) requires the highest number of hops since the distance among the nodes and the mobility of nodes are also both higher. According to the simulation results and considering a conservative value of $K$, the different values of $K$ for each scenario are (1) scenario A (A2A communication), $K=3$, (2) scenario B (A2S communication), $K=4$, (3) scenario $\mathrm{C}$ ( $\mathrm{S} 2 \mathrm{~A}$ communication), $K$ $=6$. Since the average number of hops in scenario $\mathrm{C}$ is near to 5 , it has been considered a more conservative limit in order not to affect the performance of AODV_DT_OFC.

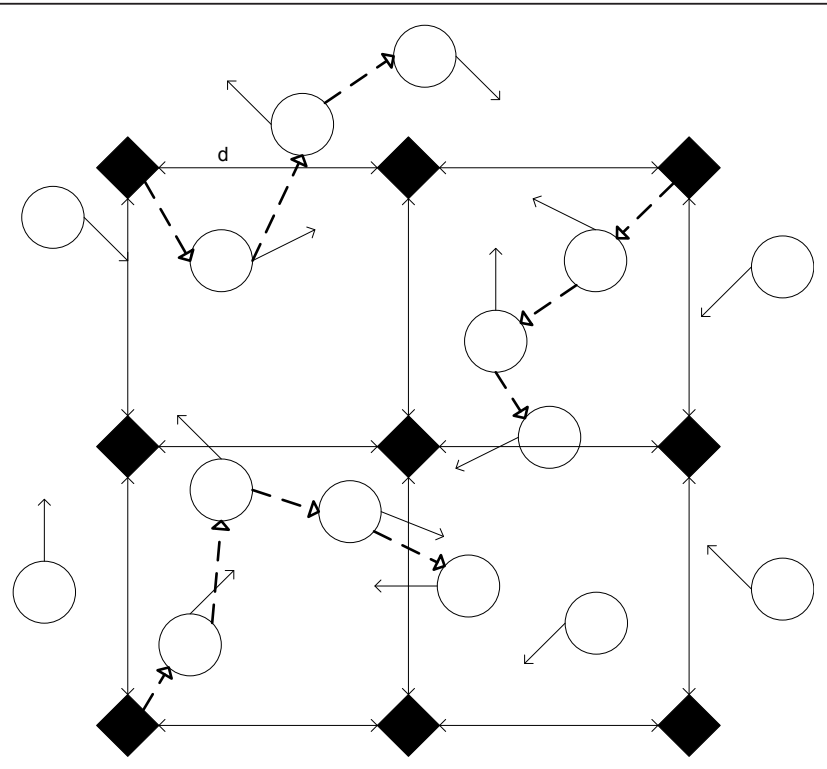

Figure 12 Sensor to actuators communications scenario. 


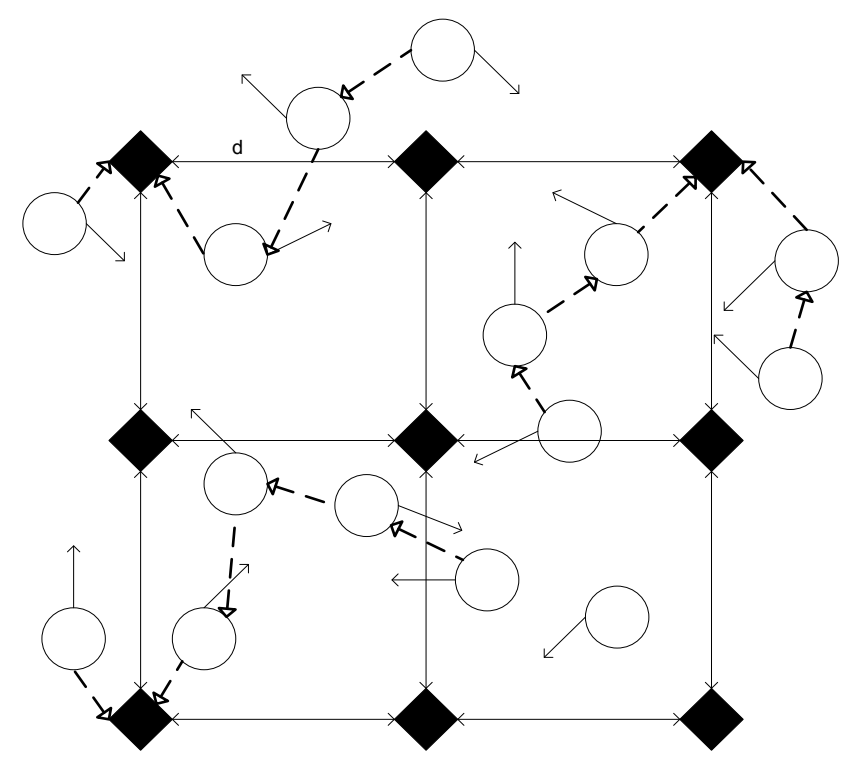

Figure 13 Average number of hops for each simulation scenario.

\subsection{Simulation results}

\subsubsection{Congestion analysis}

The proposed approaches have been compared with AODV considering the same scenarios described above. In addition, a caution zone-based scheme has also been implemented. This approach measures RRS values to define a bordering area so that nodes located in this caution zone are not taken into consideration to form new communication routes [20]. When a node receives a request packet from a node located in the caution zone, it does not forward the packet again so bordering nodes are avoided. The considered caution zone has a radius of $200 \mathrm{~m}$. As a result, nodes at distances between 200 and $250 \mathrm{~m}$ are not taken into account. The routing protocols are denoted as follows: AODV for Ad Hoc On-Demand Distance Vector, AODV_CZ for the caution zone based approach, AODV_DT for the proposed improvement of AODV with the decision tree, and AODV_DT_OFC for the proposed improvement of AODV using the decision tree and the optimized flooding control. Notice that the evaluated schemes can be divided into two types: (a) pruning schemes and (b) no

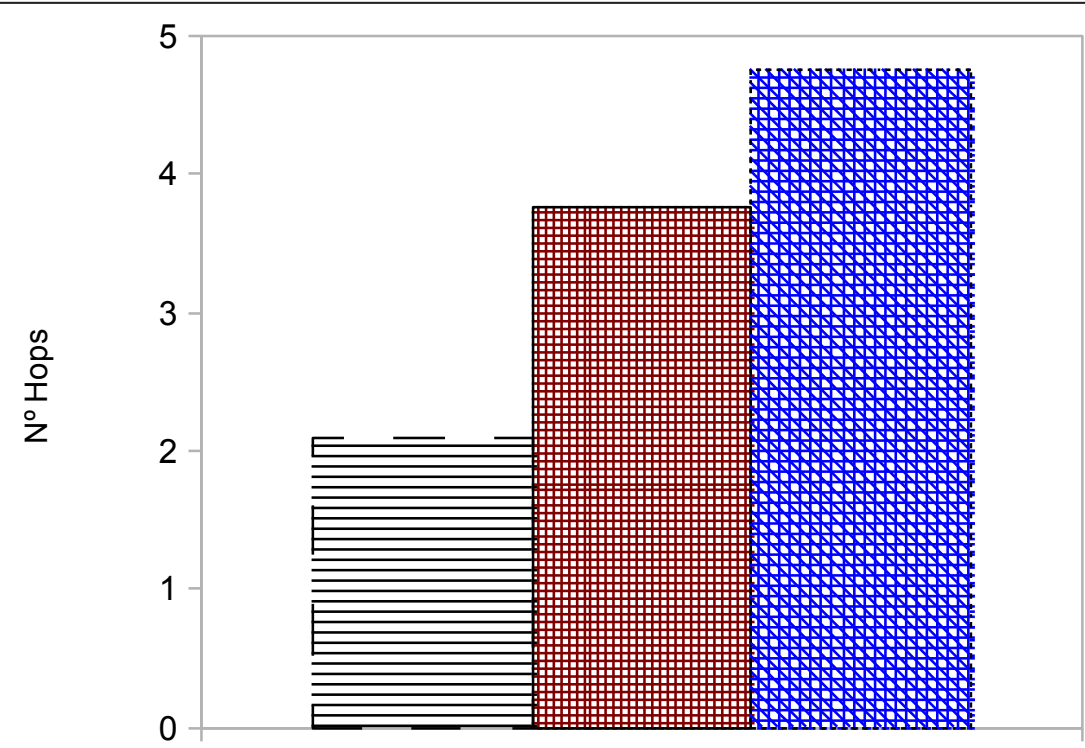

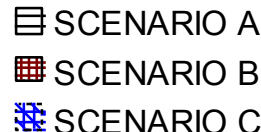

目 SCENARIO A SCENARIO C

Figure 14 General performance metrics for scenario A (A2A). (a) PDF, (b) Throughput, (c) NRL, and (d) end to end delay. 
pruning schemes. AODV_CZ and AODV_DT_OFC are both pruning schemes since they prune the broadcast operation depending on the position and mobility of nodes. On other hand, the request packets are always forwarded in both AODV and AODV_DT which are not pruning schemes. This distinction has been done in order to make a fair comparison of the routing schemes. Each point in the following graphs has been calculated by averaging out the results of five different simulations, which consider different nodes' positions, and nodes' mobility.

7.6.1.1. Scenario A: actuator-to-actuator communications (A2A) The simulation results for scenario A representing actuator-to-actuator communication are presented in Figure 15a-d. These graphs compare the general performance of the four routing protocols, namely, AODV, AODV_DT, AODV_CZ, and AODV_DT_OFC. In general, the performance of AODV_DT is similar to that of AODV. However, AODV_DT_OFC outperforms AODV as long as the number of connections among the nodes is higher than 20. Again, in terms of network throughput as shown in Figure 15b, AODV_DT_OFC exhibits a much better performance than the other three solutions, due to the least amount of network routing load used by this routing scheme. The routing loads obtained for the three schemes are compared in Figure 15c. Also, the end-to-end (E2E) delay in AODV_DT_OFC is slightly better than the other approaches as long as the number of connections is higher than 25. Although in general AODV_DT obtained better results than AODV, AODV_DT_OFC protocol consistently outperforms the other three protocols. AODV_CZ is not suitable for actuator-to-actuator communications. Notice that in the case of AODV_DT_OFC only the nodes which are going away are not taken into account to form new communication paths. This condition is less restrictive than that made by AODV_CZ. Furthermore, as the flooding control implemented in AODV_DT_OFC plays a key role in the reduction of the control packets, the amount of control packets has been reduced by $50 \%$ when the number of connections is higher than 25. This means that the power consumption of the network is notably reduced. Consequently, the lifetime of the network is extended. It is necessary to stress the paramount importance of power consumption in the design of wireless networks.

The total numbers of request packets used by each routing protocol are shown in Figure 16. The difference between AODV and AODV_DT is due to the reduction in the number of broken paths. For each broken path, a new discovery process has to be executed. The improvement observed in AODV_DT_OFC results from three factors: (1) the improvement due to the use of the decision tree which has already been observed in AODV_DT, (2) the flooding control based on mobility which avoids participation of short links in routes, and (3) the flooding control based on the number of hops which avoids useless request

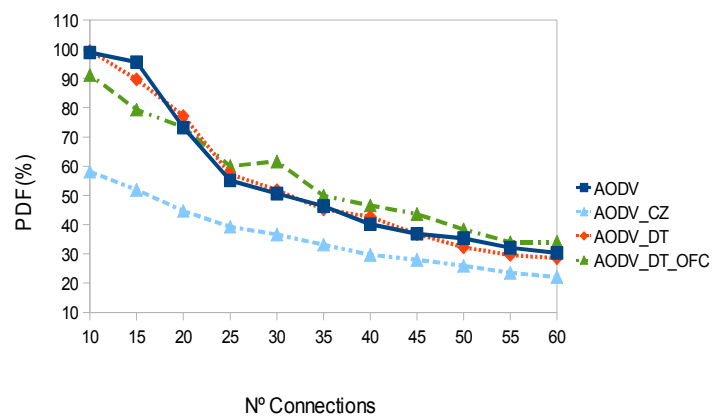

(a)

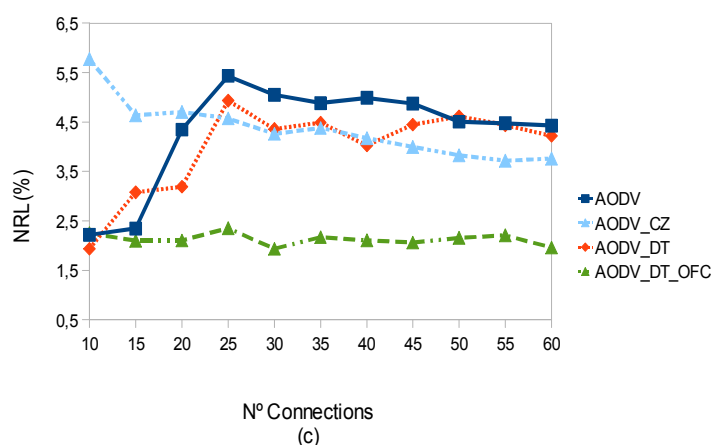

(c)

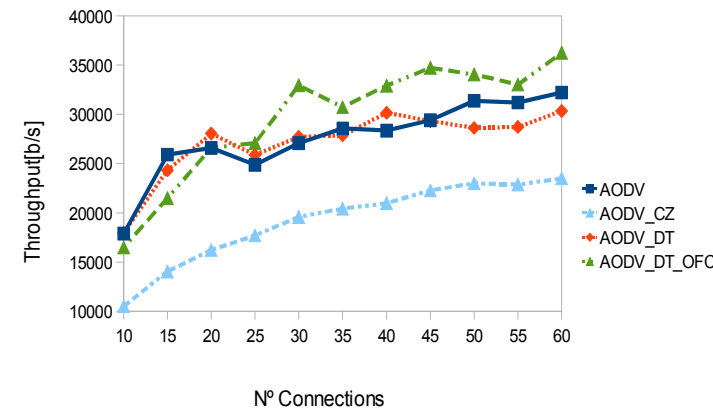

(b)

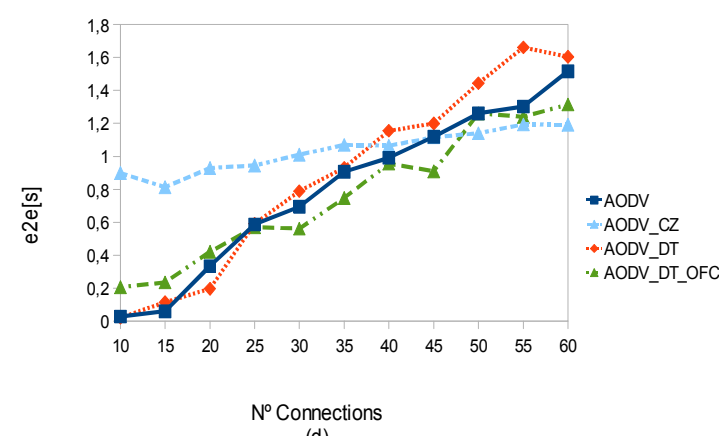

(d)

Figure 15 Total number of request packets in scenario $A(A 2 A)$ 


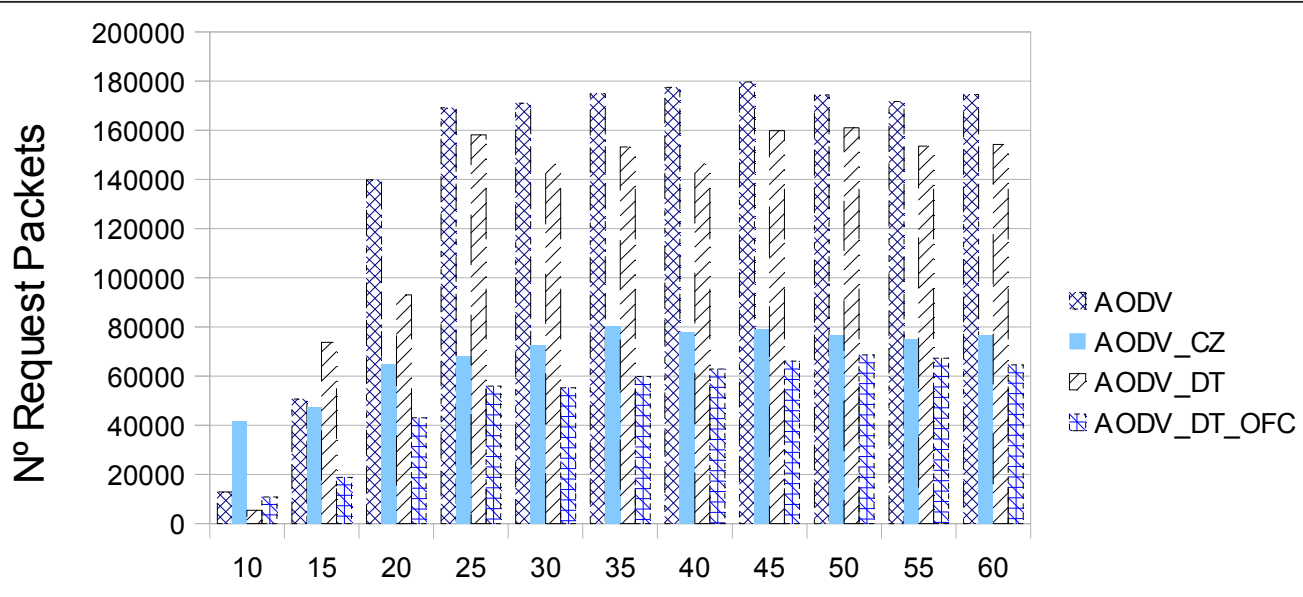

\section{$\mathrm{N}^{\circ}$ Connections}

Figure 16 Path duration metrics for scenario A (A2A). (a) Number of error packets, (b) TBE.

packets being forwarded throughout the network. The number of request packet in AODV_DT_OFC is reduced by $63 \%$. The AODV_CZ also achieves a reduction of control packets by not considering the bordering nodes.

The number of error packets is shown in Figure 17a. It is clear that the proposed AODV_DT outperforms AODV and also the proposed AODV_DT_OFC outperforms AODV_CZ. The reduction is about $32 \%$ for AODV_DT and it reaches a 63\% for AODV_DT_OFC compared with AODV. This reduction of error packets is due to the longer duration of the communication routes among the source-destination node pairs. Notice that the reduction in AODV_DT_OFC is even more accentuated since weak links will never participate in communication paths. This also happens in AODV_CZ since bordering nodes are likely to get out of the node's radio transmission range. However, the decision tree takes advantage of the other parameter such as the relative direction and the relative speed between two nodes. The number of error packets contributes to the NRL value, so it in turn benefits the global performance of the networks.

The average time between two error packets can be seen in Figure 17b. In general, the TBE is significantly longer for the proposed approaches and confirms that the proposed approaches have a longer duration of paths. The TBE is double for AODV_DT and three times higher for AODV_OFC compared with that of AODV.

7.6.1.2. Scenario B: actuator-to-sensor communications (A2S) In scenario B (A2S), the performance of AODV_DT_OFC is better in terms of PDF for a number of connections lower than 35, with an improvement of about $10 \%$ as shown in Figure 18a. Notice that the average number of hops in this scenario is higher than in scenario A (A2A). Consequently, the effect of the flooding control based on number of hops is higher since no useless request packets are used. AODV_CZ also improves the reliability of the established links by not considering bordering nodes. In this case, the performance of AODV does
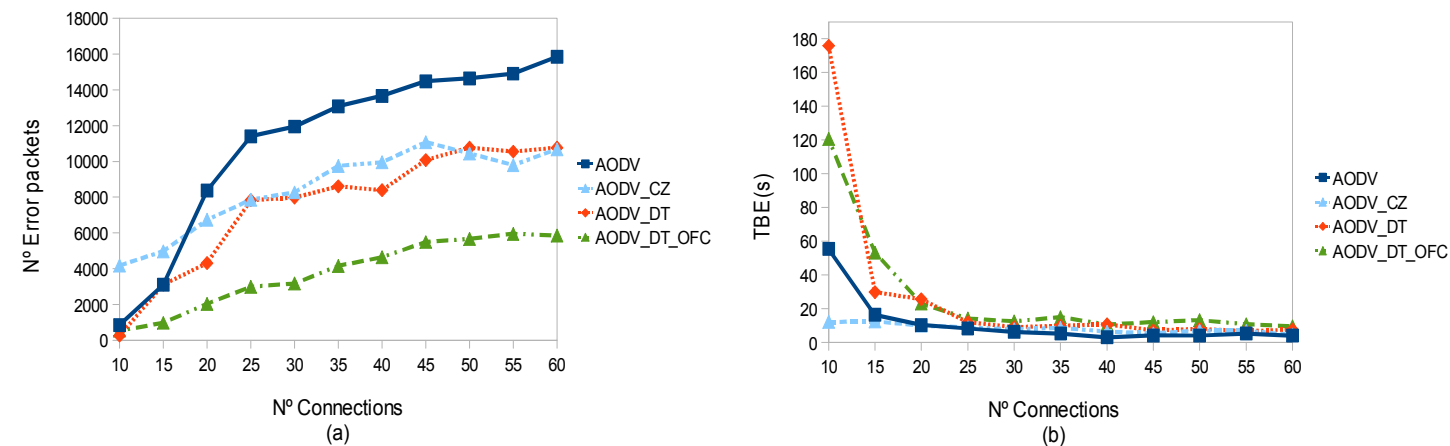

Figure 17 General Performance metrics for scenario B (A2S). (a) PDF, (b) Throughput, (c) NRL, and (d) end to end delay. 


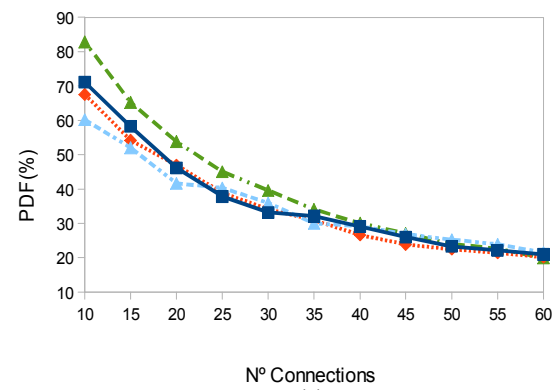

(a)

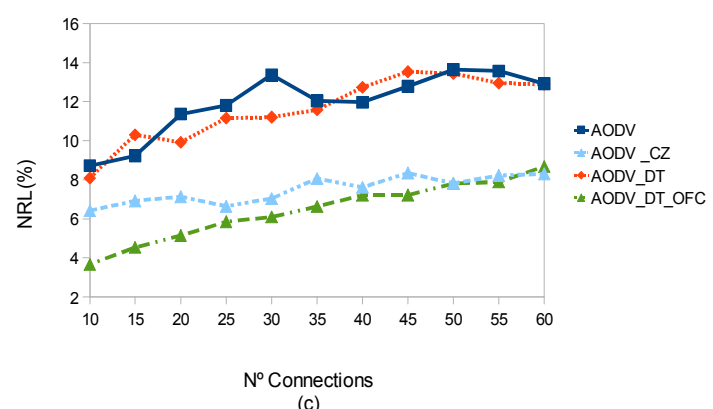

(c)

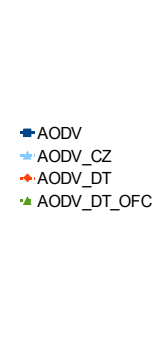

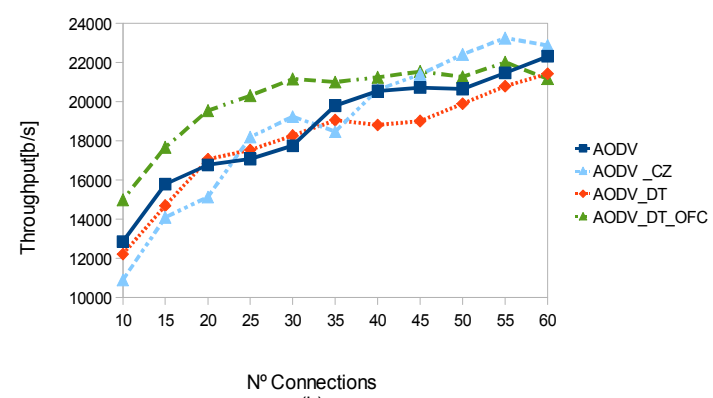

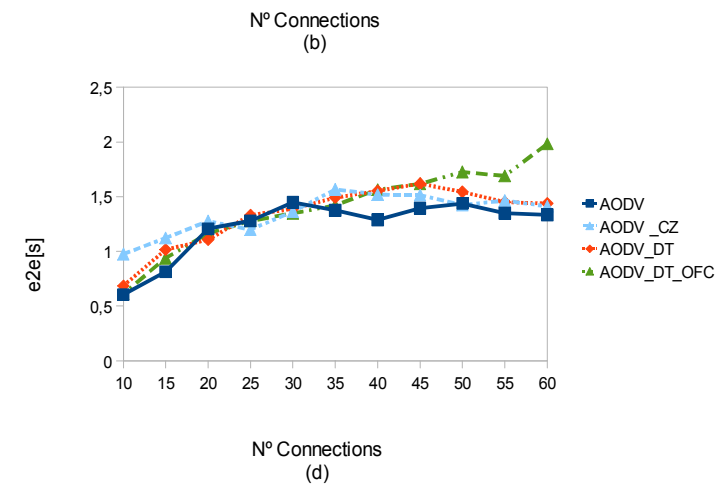

Figure 18 Total number of request packets in scenario C (S2A).

not worsen by including the caution zone. The network throughput is considerably better for AODV_DT_OFC; however, for the highest number of connections the three protocols show similar results (Figure 18b). The reason for this convergence in performance is the slightly higher end-to-end delay exhibited by AODV_DT_OFC which is represented in Figure 18c.

The proposed AODV_DT_OFC exhibits a very good performance in terms of routing load as seen in Figure 18. The amount of control packets is reduced by $50 \%$ compared with AODV. It is important to observe that the reduction of control packet does not affect the general performance of the routing protocols since only the redundant control packets are not forwarded.

The number of request packets used by each routing protocol is shown in Figure 19. The total number of request packets is reduced by $36 \%$ for AODV_DT_OFC compared with AODV, which means that the proposed flooding control is also suitable for actuator to sensor communications.

The number of error packets for scenario B (A2S) is shown in Figure 20a. The total number of error packets is reduced by $16 \%$ when AODV_DT is used and by $67 \%$ when the AODV_DT_OFC is used. As explained earlier, reduction of error packets is the main objective of the proposed approaches because of its importance in the longer duration of the established routes. The TBE packets is slightly better when AODV_DT is used and is very much better when the flooding control is added (Figure 20b).
7.6.1.3. Scenario C: sensor-to-actuator communications (S2A) The performance for scenario C (S2A) in terms of PDF, throughput, and NRL is shown in Figure 21a-c. Again the proposed schemes AODV_DT and AODV_DT_OFC achieve good performance. AODV_CZ takes more time for the selection of routes as can be seen in Figure 21d. On other hand, for higher number of connections both throughput and NRL are significantly better for AODV_DT_OFC compared with AODV.

The error packets are reduced by $25 \%$ compared with AODV when the proposed AODV_DT_OFC is used (Figure 22a). The TBE packets is again higher for the AODV_DT_OFC as can be seen in Figure 22b. Consequently, for scenario $C$, the proposed approaches also exhibit a better performance in terms of path duration.

The number of request packets used by each approach is shown in Figure 23. The number of request packets is reduced by $29 \%$ compared with AODV when the flooding control (AODV_DT_OFC) is used. It needs to be emphasized that for all the three proposed scenarios (i.e., A2A, A2S, and S2A communications), the flooding control has consistently performed well in terms of number of request packets.

From the simulation results discussed so far, it can be deduced that the decision tree has significantly improved the reliability of the selected path duration due to the reduction of error packets and the increase in TBE packets which is significantly important. With regard to the general performance of the network, the inclusion of the decision tree does not adversely affect the performance 


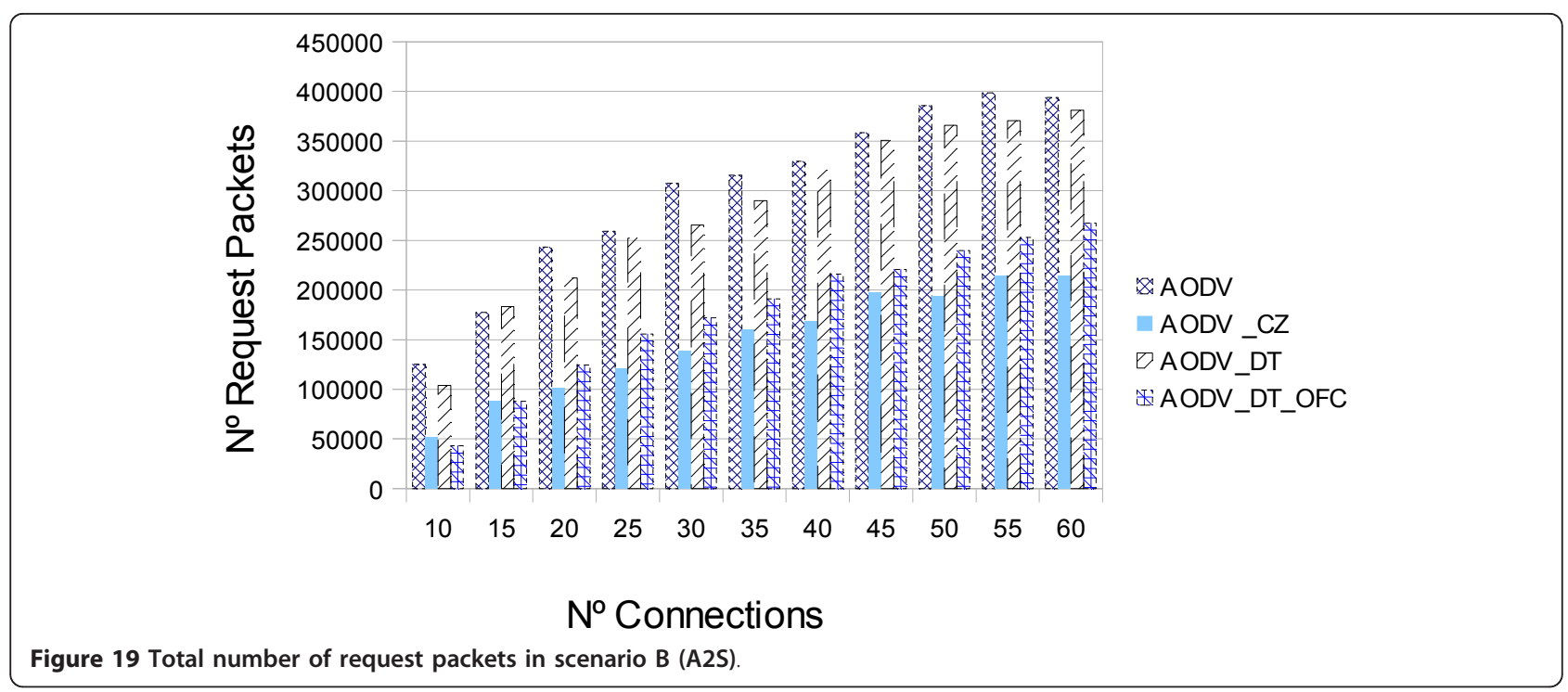

of AODV for any performance parameter. The routing load does not suffer changes because the increment of reply packets in AODV_DT is counteracted by the reduction of error packets. The flooding control in AODV_DT_OFC makes the selection of paths more reliable since any weak link is unable to participate in the formation of new routes. Moreover, the useless request packets are avoided. This efficient way of flooding control plays an important role in the improvement of the network's performance notably reducing the routing load. Furthermore, the energy consumption is significantly reduced by the decrement of routing load. Table 3 summarizes the obtained reductions in terms of request packets, error packets, and routing load. The number of connections is denoted by $N$.

Furthermore, Figures 24, 25, and 26 show the routing packets used by each approach. The comparison between AODV and AODV_DT highlights the reduction of control packets achieved by the inclusion of the decision tree for selecting stable routes. On other hand, the comparison between AODV_CZ and AODV_DT_OFC highlights the goodness of the proposed optimized flooding.

Notice that for all the considered schemes, the number of hello packets used is always the same. The proposed approaches do not modify the frequency of hello packets, they only take advantage of the Hello packets to collect positioning and speed data.

It is clear that for the three scenarios considered, the proposed approaches achieved a significant reduction of control packets compared to the other evaluated schemes. This reduction of redundant packets in turn results in the reduction of power consumption in the individual nodes.

\subsubsection{Mobility analysis}

The proposed approaches have also been compared under different mobility conditions. The node's maximum speed has been varied from 10 to $50 \mathrm{~m} / \mathrm{s}$. The

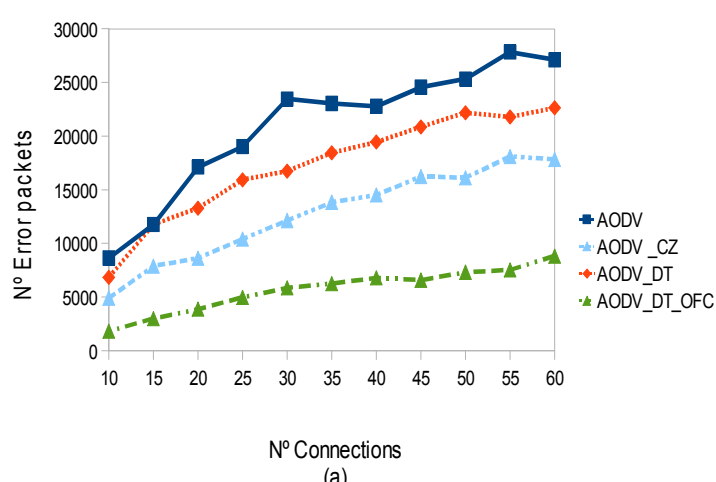

(a)

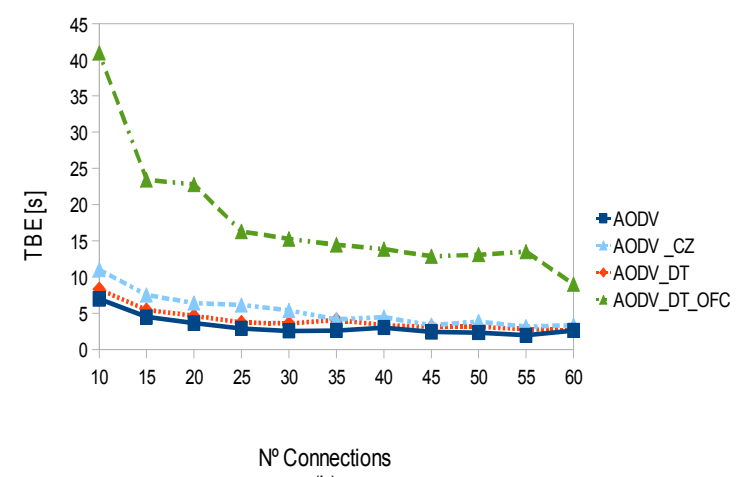

(b)

Figure 20 Path duration metrics for scenario B (A2S). (a) Number of error packets, (b) TBE. 

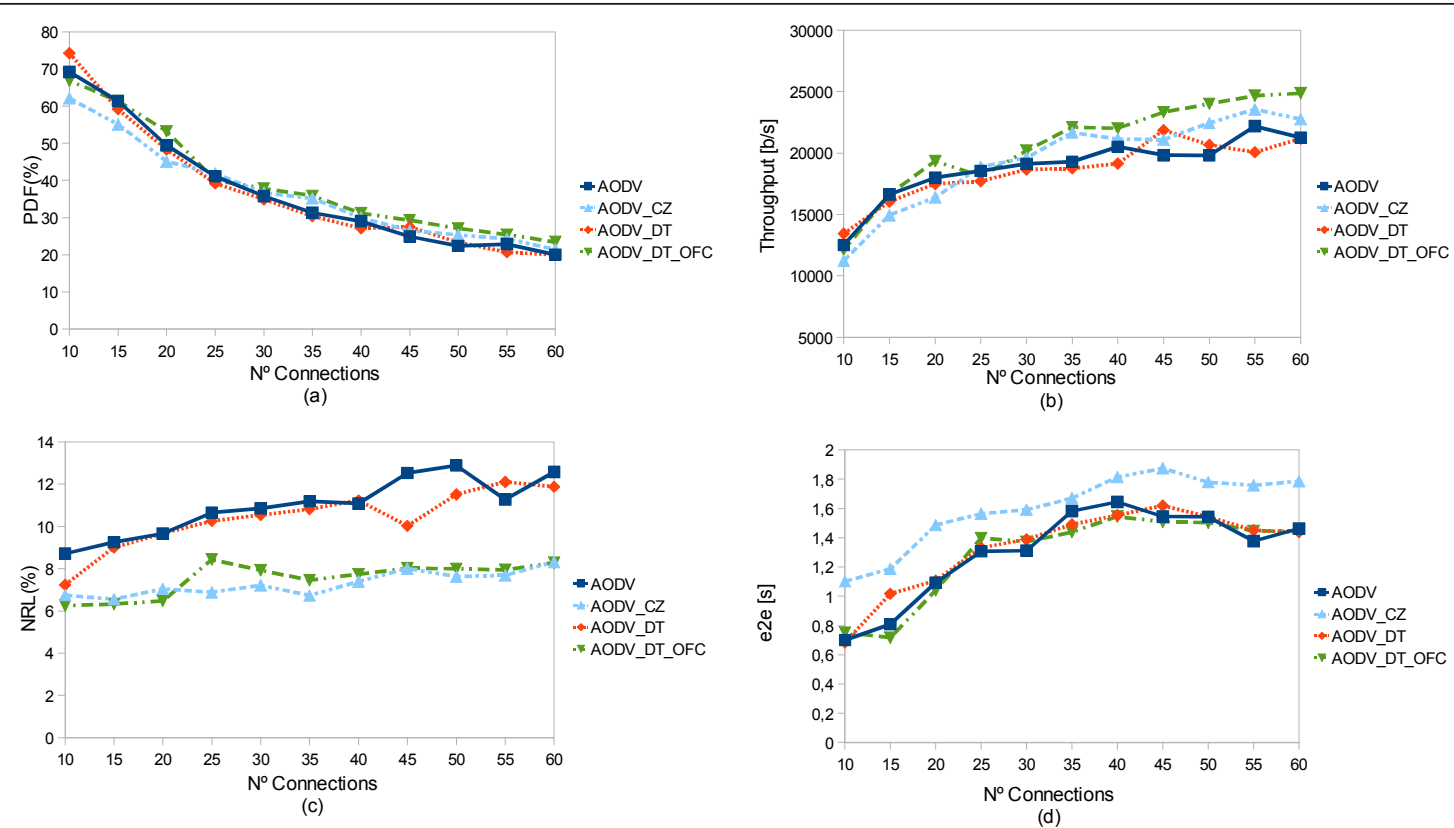

Figure 21 General performance metrics for scenario C. (a) PDF, (b) Throughput, (c) NRL, and (d) E2E.

number of connections among nodes is 30 . In this analysis, only the metrics related to path duration have been taken into account since the increase of mobility of nodes affect directly on the duration of the established communication path. The metrics considered are the number of error packets, the TBE, and the number of request packets. In addition, the NRL has also been added since it shows the global improvement at the routing layer of the proposed approaches. The proposed decision tree will be modified according to the maximum speed considered. The relative speed between two nodes will be considered as high whenever it is higher than $\frac{V_{\max }}{2}$

7.6.2.1. Scenario A: actuator-to-actuator communications (A2A) The actuator-to-actuator communications scenario is the slowest of the three proposed scenarios since both the source and the destination nodes are static nodes, and only the intermediate nodes are mobile. In this section, the proposed approaches are evaluated under different mobility of the intermediate nodes. Figure $27 \mathrm{a}, \mathrm{b}$ shows the simulation results for the scenario A, where the communications flow between two actuators. As it is shown, the proposed AODV_DT_OFC obtains the best results in terms of path duration. The decision tree improves the route selection as can be seen through the comparison between AODV and AODV_DT. The optimized flooding scheme makes use of the knowledge about the nodes directions so only the nodes going away are discarded. By contrast, in AODV_CZ any bordering node is discarded, even though it may be getting closer. Thus, AODV_CZ is

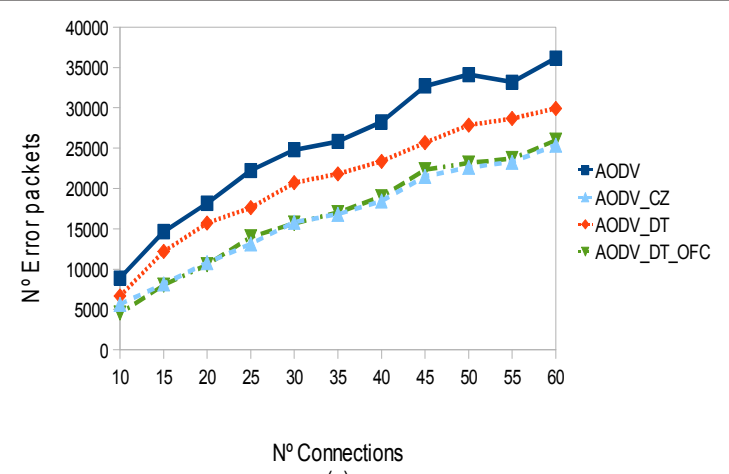

(a)

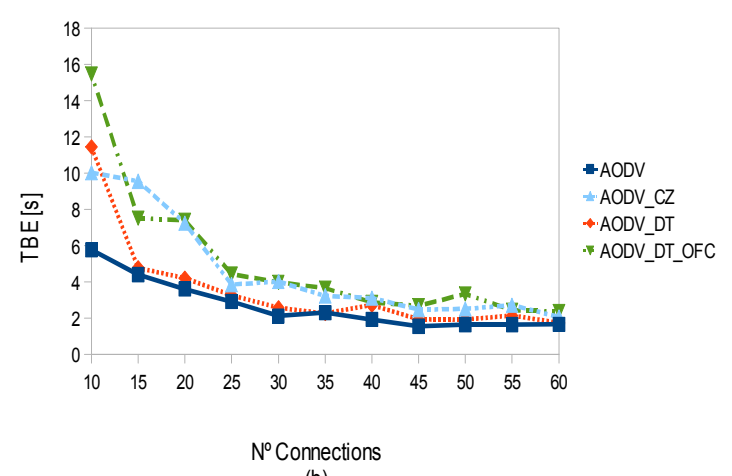

(b)

Figure 22 Path duration metrics for scenario C (S2A). (a) Number of error packets, (b) TBE. 


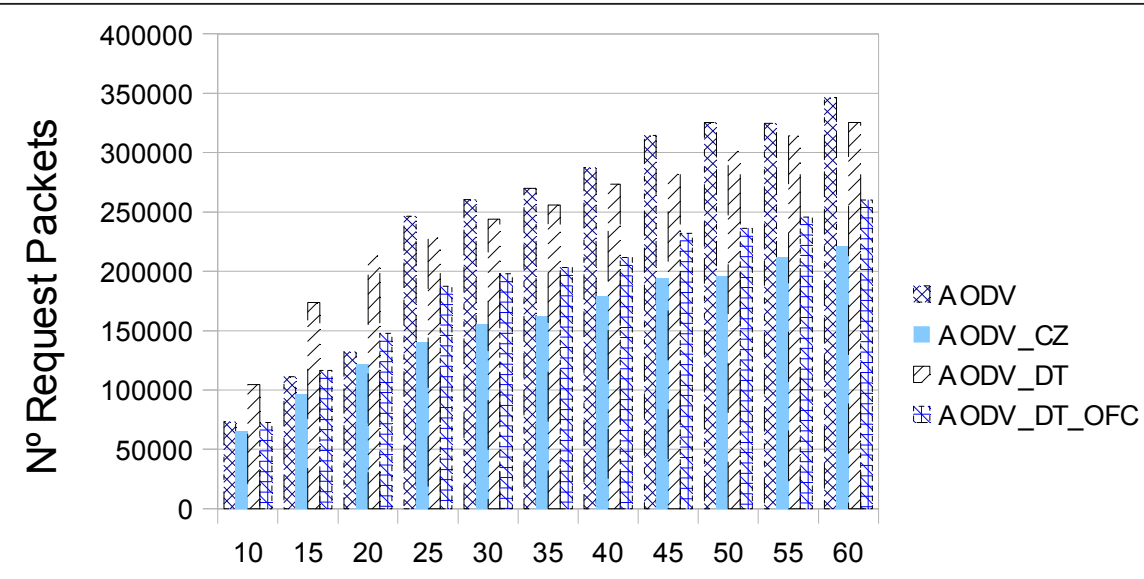

\section{$\mathrm{N}^{\circ}$ Connections}

Figure 23 Summary of control packet for scenario A (A2A). (a) AODV, (b) AODV_DT, (c) AODV_CZ, and (d) AODV_DT_OFC.

more restrictive that AODV_DT_OFC regarding with node's positions.

The proposed approaches also optimize the control packets better than AODV and AODV_CZ as can be seen in Figure 28a,b.

7.6.2.2. Scenario B: actuator-to-sensor communications (A2S) In this scenario, only the source node is static so the mobility of this scenario is higher than the previous one. The proposed AODV_DT and AODV_OFC outperform AODV and AODV_CZ, respectively, in terms of path duration, as it is shown in Figure 29a,b.

Again the proposed approaches optimize the use of control packet when the mobility of nodes is increased. The NRL and the number of request packet for actuator-to-sensor scenario are shown in Figure 30a,b.

7.6.2.3. Scenario C: sensor-to-actuator communications $(S 2 A)$ In this scenario, the mobility of nodes is the highest of the three evaluated scenarios. Only the destination node is static. Figure 31a,b shows the obtained simulation results.
With regard to the results obtained by AODV_DT and AODV_DT_OFC, the proposed decision tree also improves the route selection under high mobility conditions. The comparison of the routing schemes in terms of routing load and request packets is shown in Figure 32a,b.

\section{Conclusion}

A decision tree for path selection has been proposed in this article to improve the reliability of route selection in reactive routing protocols. Furthermore, a flooding control approach has also been proposed in order to guarantee the reliability of the selected path, and to notably reduce the amount of control packets used, which results in a considerable saving of energy. This reduction is based on an efficient way of managing flooding request packets, obtaining as a result a better performance of the network in terms of PDF and throughput. Due to the difficulty of including the theoretical models of path durations in the routing protocols, the proposed decision tree

Table 3 Summary of reductions

\begin{tabular}{|c|c|c|c|c|c|c|}
\hline \multirow[t]{2}{*}{ Parameters } & \multicolumn{2}{|c|}{ A2A (scenario A) } & \multicolumn{2}{|c|}{ A2S (scenario B) } & \multicolumn{2}{|c|}{ S2A (scenario C) } \\
\hline & AODV_DT & AODV_DT_OFC & AODV_DT & AODV_DT_OFC & AODV_DT & AODV_DT_OFC \\
\hline Request & $11 \%$ & $63 \%$ & $7 \%$ & $36 \%$ & $10 \%$ & $29 \%$ \\
\hline packets & $N=60$ & $N=60$ & $N=55$ & $N=55$ & $N=45$ & $N=50$ \\
\hline \multicolumn{7}{|l|}{ (RREQs) } \\
\hline \multicolumn{7}{|c|}{ (maximum values) } \\
\hline Error & $32 \%$ & $63 \%$ & $16 \%$ & $67 \%$ & Up to 18 & $25 \%$ \\
\hline packets & $N>15$ & $N>15$ & $N>15$ & $N>15$ & $\%$ & All range \\
\hline (RERRs) & & & & & All range & \\
\hline Routing & $22 \%$ & $50 \%$ & - & $50 \%$ & - & $27 \%$ \\
\hline load & $15<N<$ & $N>20$ & & All range & & All range \\
\hline (NRL) & 45 & & & & & \\
\hline
\end{tabular}



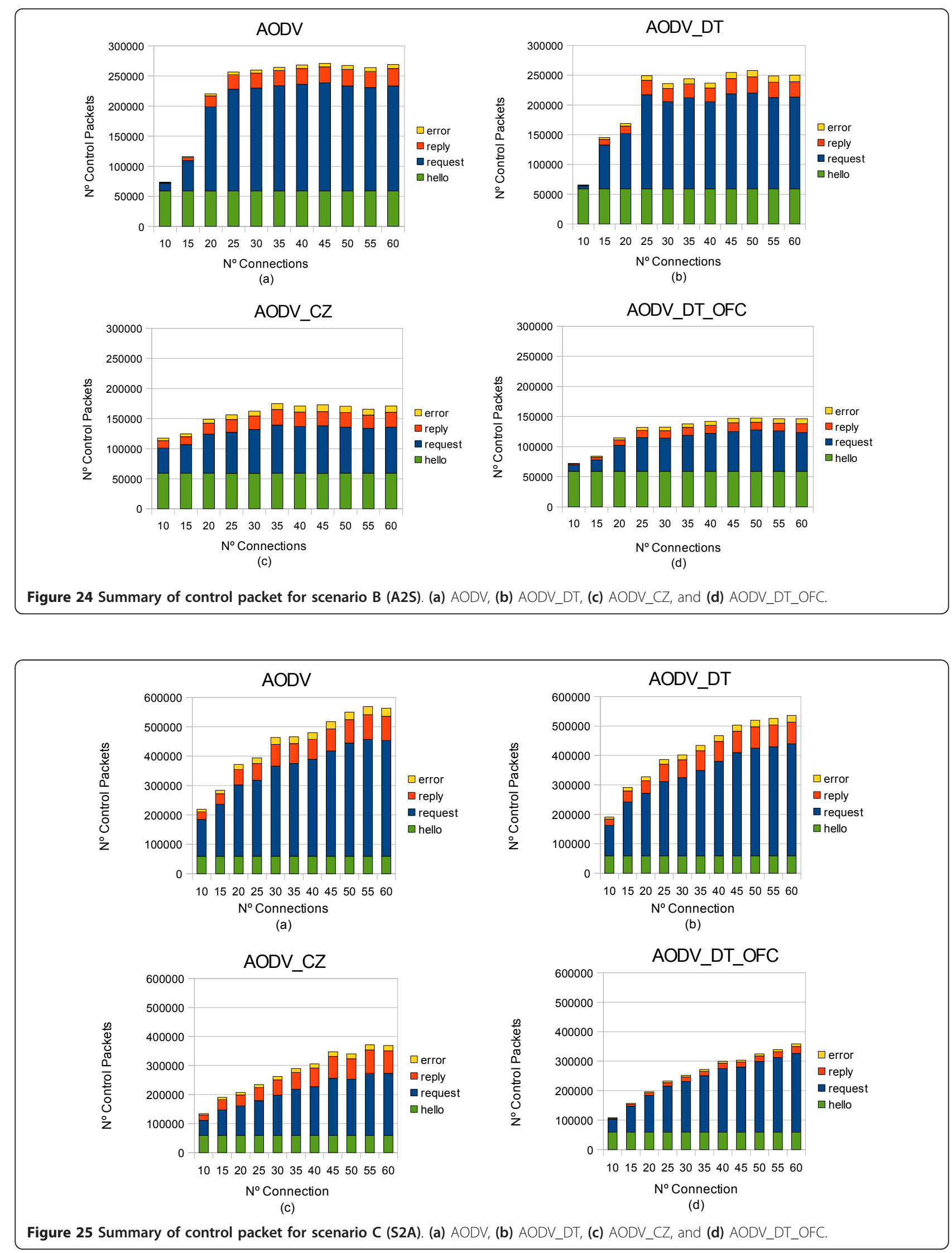


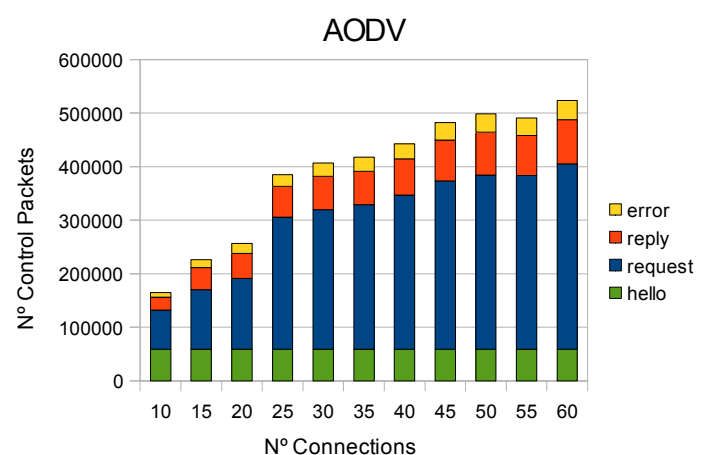

(a)

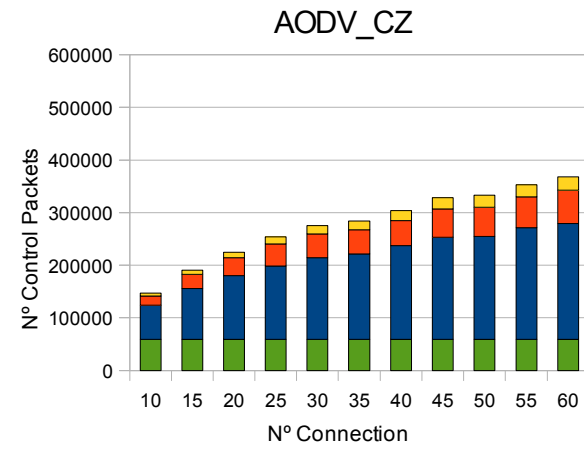

(c)

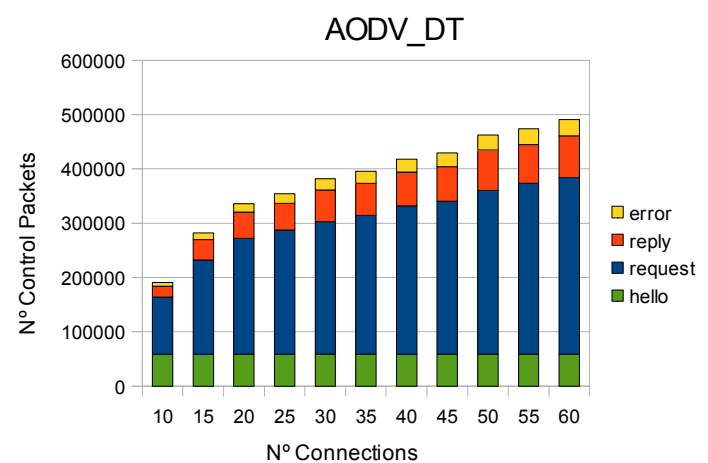

(b)

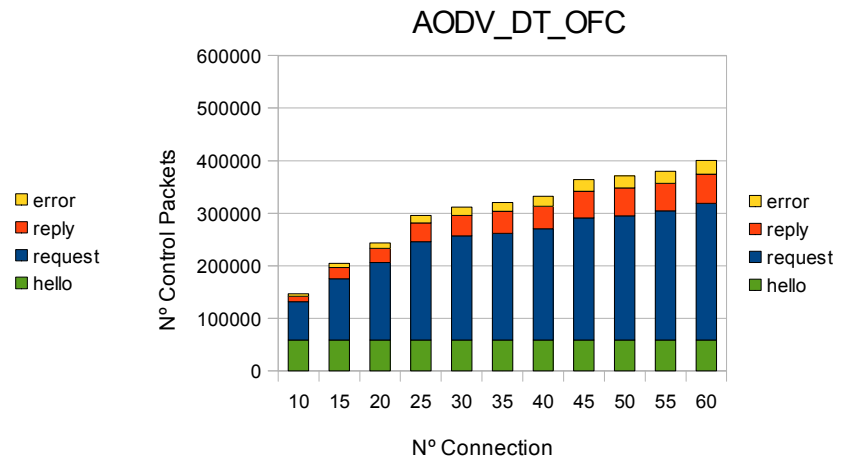

(d)

Figure 26 Path duration metrics for scenario A (A2A) under different mobility conditions. (a) Number of error packets, (b) TBE.

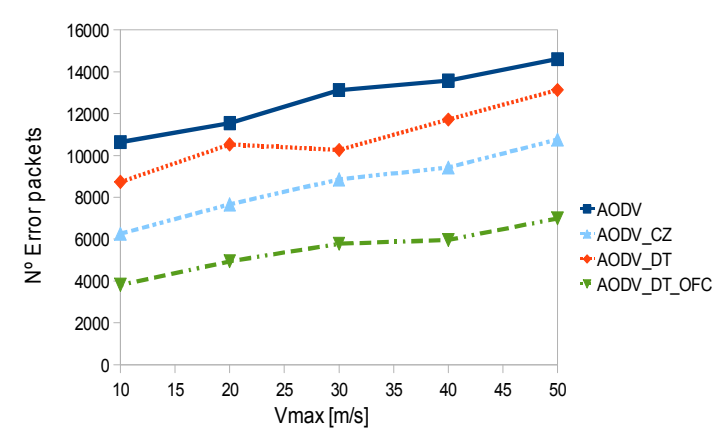

(a)

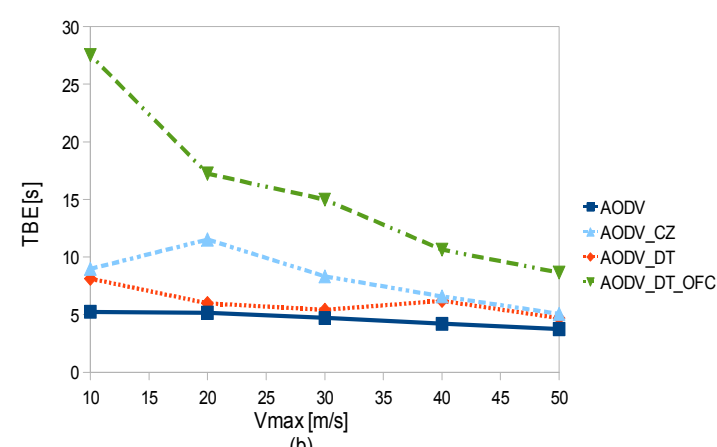

(b)

Figure 27 Simulation results for scenario A (A2A) under different mobility conditions. (a) NRL, (b) number of request packets.

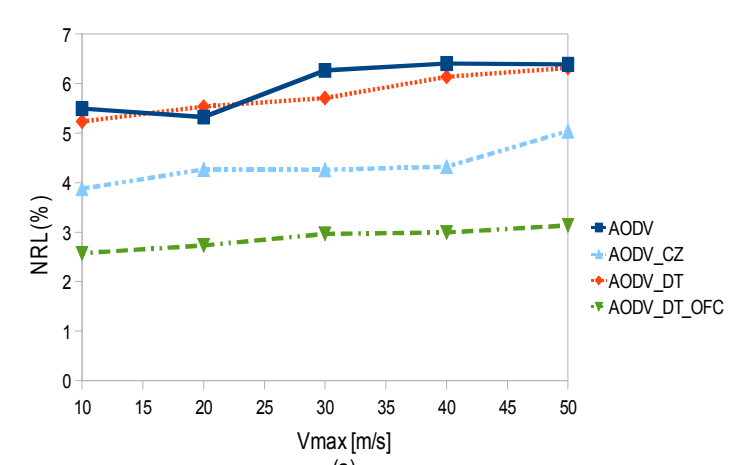

(a)

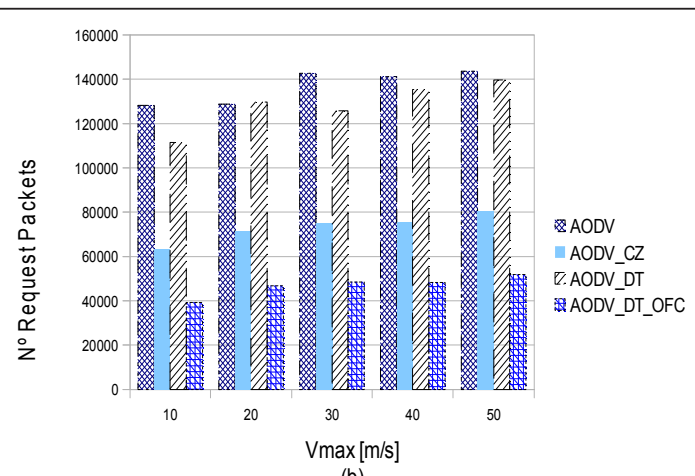

(b)

Figure 28 Path duration metrics for scenario B (A2S) under different mobility conditions. (a) Number of error packets, (b) TBE. 


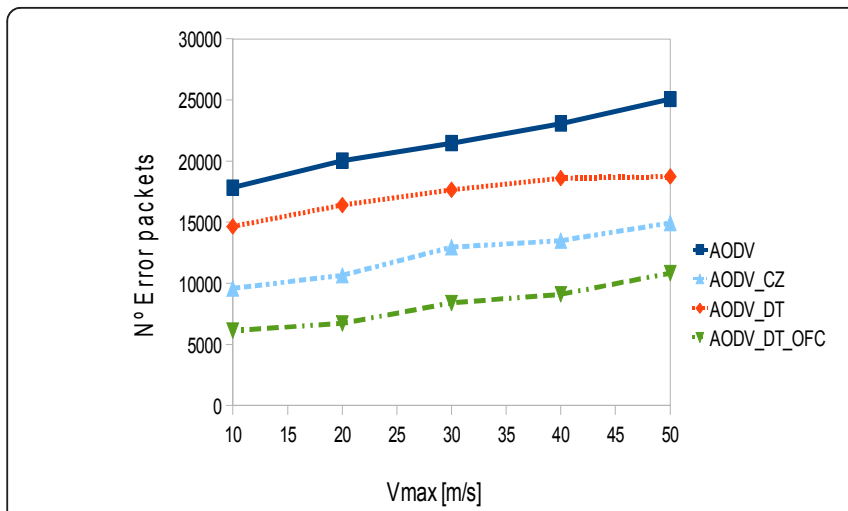

(a)

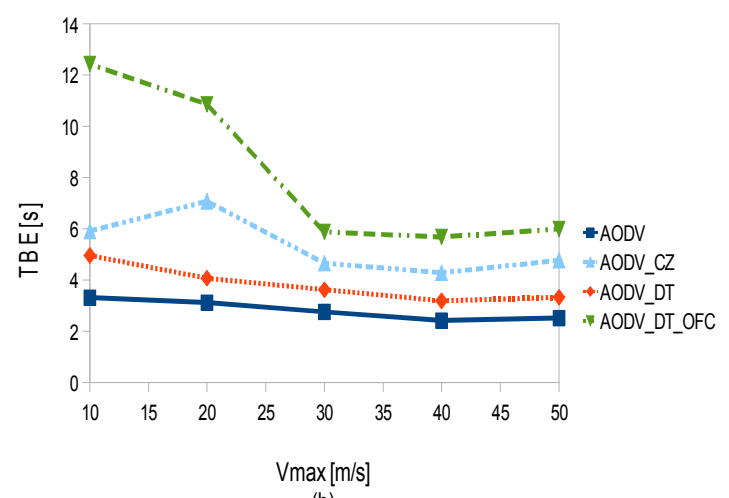

(b)

Figure 29 Simulation results for scenario B (A2S) under different mobility conditions. (a) NRL, (b) number of request packets.

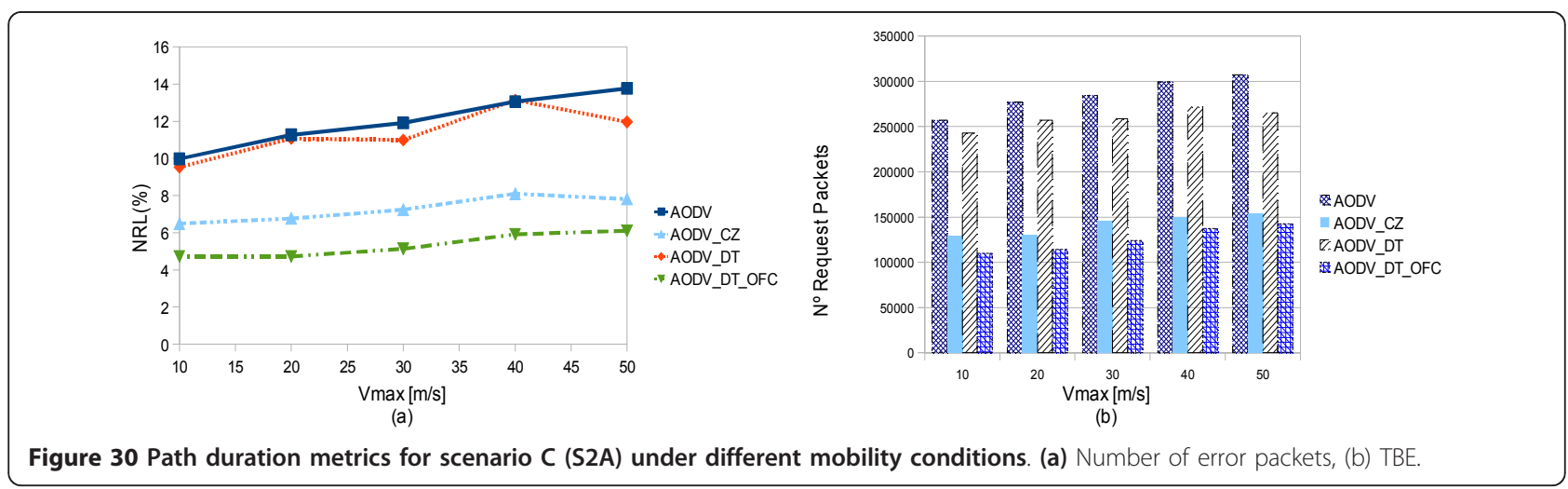

simplifies route selection by summarizing several assumptions related to route duration and helping to select long-life routes. To carry out the proposed improvements over AODV routing protocol, several modifications have been done on the route discovery procedure. The proposed approaches have been tested and compared to AODV considering three representative connectivity scenarios in WSANs. Each scenario requires different number of hops to establish the communication path between source and destination nodes. Simulation results have shown that when the decision tree is used the number of error packets is reduced. Furthermore, if the flooding control is added the reductions are much more significant. When the flooding control is used the control packets are notably reduced; thanks to an effective reduction of useless request packets, leading to a reduction in routing load and energy consumption. This reduction is of vital importance since it helps to extend

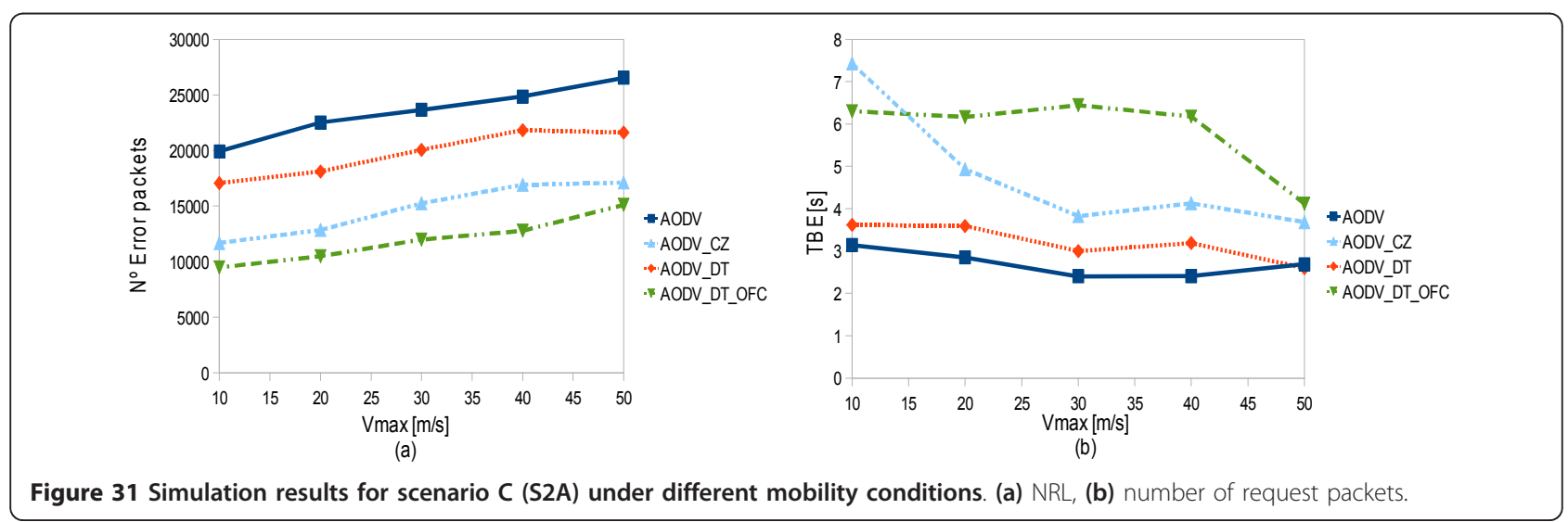




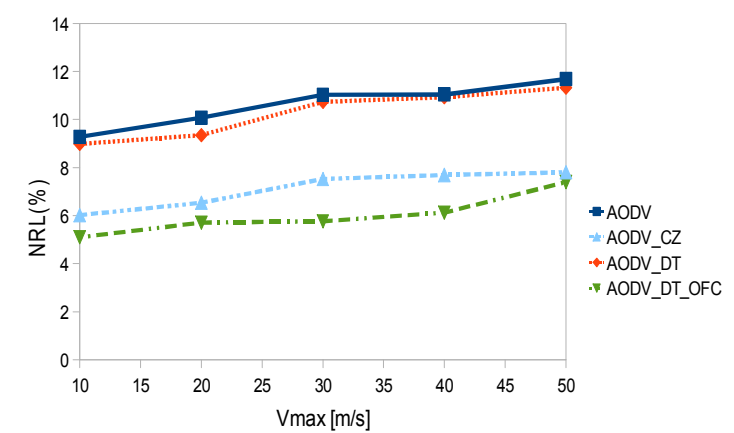

(a)

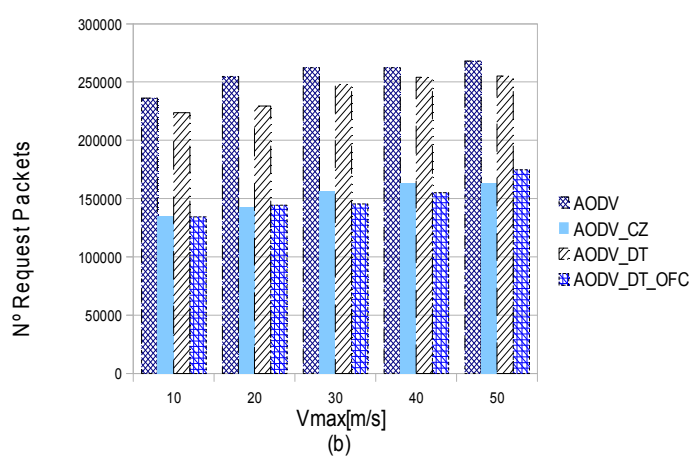

Figure 32 Flooding control based on mobility factors

the lifetime of wireless networks routes. The proposed approaches have been evaluated under different congestion and mobility conditions. Both analyses have demonstrated the validity of the proposed decision tree for improving path selection. As an extension of this research in future, it would be interesting to evaluate the proposed approaches under different mobility models for example, Manhattan grid mobility model and Random Direction model. In addition, it would be useful to take into account shorter movements of the actuators around their positions for the simulation.

\section{Acknowledgements}

The authors would like to thank the Spanish Government, the Junta de Andalucía and the European Union (FEDER funds) for their financial support $(\mathrm{R}+\mathrm{D}$ projects under reference P10-TEP-5791 and DPI2009-07955).

\section{Competing interests}

The authors declare that they have no competing interests.

\section{Author details}

${ }^{1} E$. S. Ingenieros, University of Seville, Avda. Camino de los Descubrimientos $s / n, 41092$, Seville, Spain ${ }^{2}$ Liverpool John Moores University, Liverpool, England, UK

Received: 25 July 2011 Accepted: 19 April 2012 Published: 19 April 2012

\section{References}

1. R Verdone, D Dardani, G Mazzini, A Conti, Wireless Sensor and Actuator Networks (Elsevier, 2008)

2. C Jimming, C Xianghui, C Peng, Y Xiao, S Youxian, Distributed collaborative control for industrial automation with wireless sensor and actuator networks. IEEE Trans Ind Electron. 57(12), 4219-4230 (2010)

3. D Culler, D Estrin, M Srivastava, Overview of sensor networks. IEEE Comput. 37(8), 41-49 (2004)

4. K Akkaya, M Younis, A survey on routing protocols for wireless sensor networks. Ad Hoc Netw. 3, 325-349 (2003)

5. R Beraldi, R Baldoni, in Unicast Routing Techniques for Mobile Ad Hoc Networks, ed. by Mohammad Llyas (The Handbook of Ad Hoc Networks Electrical Engineering Handbook Series, 2003)

6. S Mohseni, R Hassan, A Patel, R Razali, Comparative review study of reactive and proactive routing protocols in MANETs, in Paper presented at 4th IEEE International Conference on Digital Ecosystems and Technologies (IEEE DEST 2010), Dubai, pp. 304-309 (2010)

7. L Layuan, $L$ Chunlin, $Y$ Peian, Performance evaluation and simulations of routing protocols in ad hoc networks. Comput Commun. 30(8), 1890-1898 (2007)
8. NSM Usop, A Abdullah, AFA Abidin, Performance evaluation of AODV, DSDV \& DSR routing protocol in grid environment. IJCSNS Int J Comput Sci Netw Secur. 9(7), 261-268 (2009)

9. C Maihofer, A survey of geocast routing protocols. IEEE Commun Surv Tutor. 6(2), 32-42 (2004)

10. N Patwari, JN Ash, S Kyperountas, AO Hero III, RL Moses, NS Correal, Locating the nodes. Cooperative localization in wireless sensor networks. Signal Process Mag. 54, 54-69 (2005)

11. K Young-Bae, V Nitin, Location-aided routing (LAR) in mobile ad hoc networks. Wirel Netw. 6, 307-321 (2000)

12. S Young-Joo, K Won-Ik, K Dong-Hee, in GPS-Based Reliable Routing Algorithms for Ad Hoc Networks, ed. by Mohammad Llyas The Handbook of Ad Hoc Networks Electrical Engineering Handbook Series, (2003)

13. C Ruay-Shiung, L Shing-Jiuan, Long-lived path routing with received signal strength for ad hoc networks. in Paper presented at 1st International Symposium on Wireless Pervasive Computing, Xinjiang 1-6 (2006)

14. KM Mahesh, SR Das, Ad hoc on-demand multipath distance vector routing. Wirel Commun Mob Comput. 6(3), 969-988 (2006)

15. J Broch, DA Maltz, DB Johson, Y Hu, J Jetcheva, A performance comparison of multi-hop wireless ad hoc networks routing protocols, in Proceedings of the Fourth Annual ACM/IEEE International Conference on Mobile Computing and Networking (MobiCom'98), New York, 1998, pp. 1-13

16. L Hanzo, R Tafazolli, A survey of QoS routing solutions for mobile ad hoc networks. IEEE Commun Surv Tutor. 9(2), 50-70 (2007)

17. A Alshanyour, $\cup$ Baroundi, A simulation study: the impact of random and realistic mobility models on the performance of Bypass-AODV in ad hoc wireless networks. EURASIP J Wirel Commun Netw. 2010, 293370 (2010)

18. I Rubin, C Liu, Link stability models for QoS ad hoc routing algorithms, in Paper presented at IEEE 58th Vehicular Technology Conference, vol. 5. (Florida, 2003), pp. 3084-3088

19. R Shah, J Rabaey, Energy aware routing for low energy ad hoc sensor networks, in Proceedings of IEEE Wireless Communications and Networking Conference (WCNC), vol. 1. Florida, pp. 350-355 (2002)

20. S Krco, M Ducipnov, Improved neighbor detection algorithm for AODV routing protocol. IEEE Commun Lett. 7(12), 584-586 (2003)

21. G Ferrari, SA Malvassori, OK Tonguz, On physical-oriented routing with power control in ad hoc wireless networks. IET Commun. 2(2), 306-319 (2008)

22. T Nadeem, S Parthasarathy, Mobility control for throughput maximization in ad hoc networks. Wirel Commun Mob Comput. 6, 951-967 (2006)

23. T-C Tsai, S-T Tsai, A cross-layer design routing design for multi-interface wireless mesh networks. EURASIP J Wirel Commun Netw. 2009, 208524 (2009)

24. C Wei-Ho, Probabilistic analysis of routes on mobile ad hoc networks. IEEE Commun Lett. 8(8), 506-508 (2004)

25. J Lee, Y Su, C Shen, A comparative study of wireless protocols: Bluetooth, UWB, Zigbee, and Wi-Fi, in Paper presented at the 33rd Annual Conference of IEEE Industrial Electronics Society (IECON), Taipei, 2007, pp. 5-8

26. CE Perkins, EM Royer, Ad-hoc on-demand distance vector routing, in Proceeding of IEEE Workshop on Mobile Computing Systems and Applications (WMCSA), New Orleans, pp. 1-11 (1999) 
27. Y Dan, L Hui, Path availability in ad hoc networks, in Paper presented at 10th International Conference on Telecommunications (ICT 2003), vol. 1. Tahiti, pp. 383-387 (2003)

28. M Pascoe-Chalke, J Gomez, V Rangel, M López-Guerrero, Route duration modelling for mobile ad hoc networks. Wirel Netw. 16(3), 743-757 (2009)

29. F Bai, N Sadagopan, B Krisgnamachari, A Helmy, Modeling path duration distributions in MANETs and their impact on reactive routing protocols. IEEE J Sel Areas Commun. 22(7), 1357-1373 (2004)

30. DB Jonshon, DA Maltz, J Broch, in DSR: The Dynamic Source Routing Protocol for Multi-Hop Wireless Ad Hoc Networks, ed. by Charles E (Perkins, Ad Hoc Networking Addison-Wesley, 2001), pp. 139-172

31. Y Khamayseh, OM Darwish, SA Wedian, MA-AODV, mobility aware routing protocols for mobile ad hoc networks, in Paper presented at Fourth International Conference on Systems and Networks Communications (ICSNC '09), Porto, pp. 25-29 (2009)

32. B Ramachandran, S Shanmugavel, Impact of node density on cross layer design for reliable route discovery in mobile ad-hoc networks. Ubiquitous Comput Commun J. 3, 17-23 (2008)

33. PL Yang, GC Qin, H Wang, L Zhang, G Chen, MOTOROLA, MObility, TOlerable ROute seLection algorithms in wireless networks. IET Commun. 4(7), 837-849 (2010)

34. SK Dhueandher, S Misra, MS Obaidar, M Gupta, K Diwakar, P Gupta, Efficient angular routing protocol for inter-vehicular communication in vehicular ad hoc networks. IET Commun. 4(7), 826-836 (2004)

35. Y Han, RJ La, Maximizing path durations in mobile ad hoc networks, in Paper presented at 40th Annual Conference on Information Sciences and Systems, Princenton, pp. 26-31 (2006)

36. Y Han, RJ La, Distribution of path durations in mobile ad hoc networks and path selection. IEEE Trans Netw. 15(5), 993-1006 (2007)

37. P Gupta, PR Kumar, The capacity of wireless networks. IEEE Transaction on Inf Theory. 46(2), 388-403 (2000)

38. CE Perkins, P Bhagwat, Highly dynamic destination-sequenced distancevector routing (DSDV) for mobile computers. Comput Commun. 24 234-244 (1994)

39. L Weng, J Yang, A cross-layer design stability based routing mechanism for ultra wideband networks. Comput Commun. 33(18), 2185-2194 (2010)

40. K Bhargavan, D Obradovic, CA Gutner, Formal verification of standards for distance vector routing protocols. J ACM. 49(4), 538-576 (2002)

41. K Fall, K Varadhan, The ns Manual (formerly ns Notes and Documentation). http://www.isi.edu/nsnam/ns/ns-documentation.html (2010). Accessed 22 July 2011

42. T Camp, J Boleng, V Davies, A survey of mobility models for ad hoc network research. Wirel Commun Mob Comput. 2(5), 483-502 (2002)

doi:10.1186/1687-1499-2012-147

Cite this article as: Reina et al:: Route duration improvement in wireless sensor and actuator networks based on mobility parameters and flooding control. EURASIP Journal on Wireless Communications and Networking 2012 2012:147.

\section{Submit your manuscript to a SpringerOpen ${ }^{\circ}$ journal and benefit from:}

- Convenient online submission

- Rigorous peer review

- Immediate publication on acceptance

- Open access: articles freely available online

- High visibility within the field

- Retaining the copyright to your article

Submit your next manuscript at $\gg$ springeropen.com 